\title{
Stochastic Ratcheting on a Funneled Energy Landscape Is Necessary for Highly Efficient Contractility of Actomyosin Force Dipoles
}

\author{
James E. Komianos and Garegin A. Papoian* \\ Department of Chemistry and Biochemistry, University of Maryland, College Park, Maryland 20742, USA \\ and Institute for Physical Science and Technology, University of Maryland, \\ College Park, Maryland 20742, USA
}

(Received 15 June 2017; revised manuscript received 5 December 2017; published 4 April 2018)

\begin{abstract}
Current understanding of how contractility emerges in disordered actomyosin networks of nonmuscle cells is still largely based on the intuition derived from earlier works on muscle contractility. In addition, in disordered networks, passive cross-linkers have been hypothesized to percolate force chains in the network, hence, establishing large-scale connectivity between local contractile clusters. This view, however, largely overlooks the free energy of cross-linker binding at the microscale, which, even in the absence of active fluctuations, provides a thermodynamic drive towards highly overlapping filamentous states. In this work, we use stochastic simulations and mean-field theory to shed light on the dynamics of a single actomyosin force dipole - a pair of antiparallel actin filaments interacting with active myosin II motors and passive cross-linkers. We first show that while passive cross-linking without motor activity can produce significant contraction between a pair of actin filaments, driven by thermodynamic favorability of cross-linker binding, a sharp onset of kinetic arrest exists at large cross-link binding energies, greatly diminishing the effectiveness of this contractility mechanism. Then, when considering an active force dipole containing nonmuscle myosin II, we find that cross-linkers can also serve as a structural ratchet when the motor dissociates stochastically from the actin filaments, resulting in significant force amplification when both molecules are present. Our results provide predictions of how actomyosin force dipoles behave at the molecular level with respect to filament boundary conditions, passive cross-linking, and motor activity, which can explicitly be tested using an optical trapping experiment.
\end{abstract}

DOI: 10.1103/PhysRevX.8.021006

\section{INTRODUCTION}

Many eukaryotic cells rely on the ability to reshape and reform their interior cytoskeletal polymer structure to produce directed motion [1]. This biological active matter, which constantly consumes and dissipates energy in its surrounding environment, exerts directional forces allowing the cell to dynamically respond to a variety of chemical or mechanical extracellular cues [2-4]. An intriguing example of a cytoskeletal system that drives these cellular morphological changes is the cell's actomyosin network-a polymeric network of long, thin actin fibers which can be nucleated and rearranged via actin binding proteins and active, adenosine triphosphate (ATP)-consuming molecular motors to produce cellular forces in a variety of

\footnotetext{
*gpapoian@umd.edu
}

Published by the American Physical Society under the terms of the Creative Commons Attribution 4.0 International license. Further distribution of this work must maintain attribution to the author(s) and the published article's title, journal citation, and DOI.
Subject Areas: Biological Physics, Soft Matter, Statistical Physics structures, providing a dynamic scaffold for the cell body. The distinct ability of the combination of actin filaments, passive actin cross-linking proteins, and myosin II molecular motors to produce contractile cellular force is of fundamental importance in many cell types and has been well studied in muscle cells [5-7], where parallel arrangements of filaments in opposite polarities allows for a directed, inward pull of the cell's sarcomeric unit (Fig. 1).

Contractility is, however, much less understood in motile nonmuscle cells, where it frequently emerges from an isotropic filament network with little geometric or polar ordering, indicating some type of a spontaneous symmetrybreaking process $[9,10]$. These contractile networks are responsible for diverse microstructural dynamics, including lamellar stress fiber formation of tens of bundled filaments [shown in Fig. 1(b)], cell rear retraction that is essential to locomotion, and tension maintenance in the thin submembrane cortical layer surrounding the cell [11]. Prior works on explaining the emergence of contractility in disordered actomyosin networks have pointed to a variety of effects, including the potential importance of filament buckling [12-15], actin filament treadmilling [16,17], and the role of 


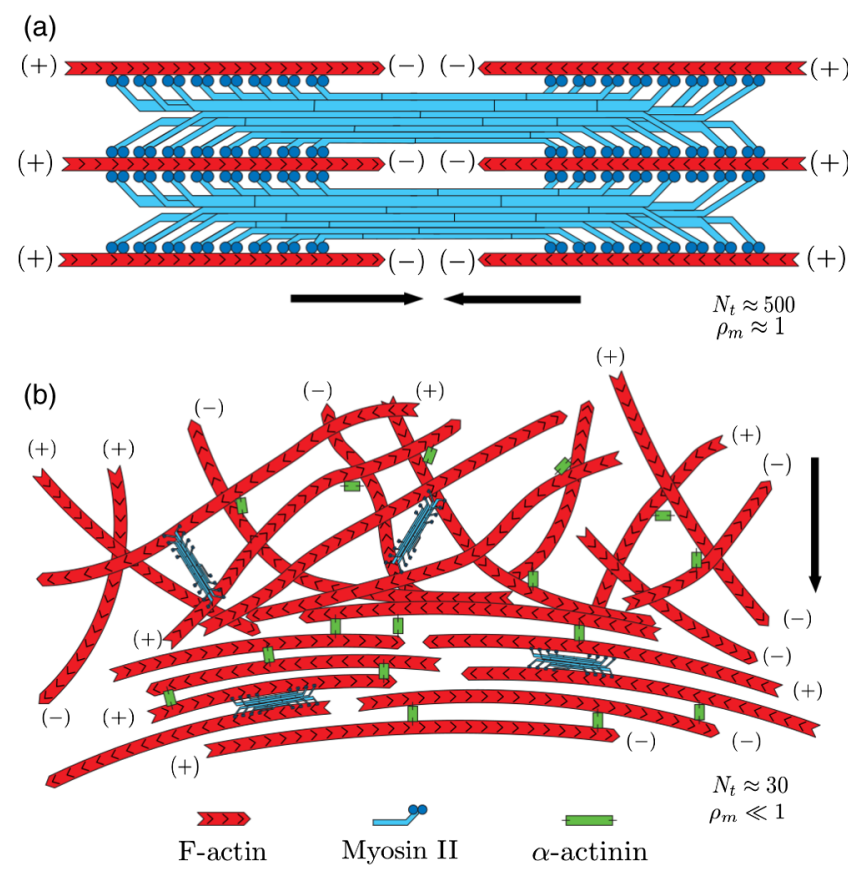

FIG. 1. Actomyosin contractility mechanisms in a variety of cell types. (a) In a muscle sarcomere, filamentous actin ( $F$-actin) are aligned in bands of opposite polarity such that a bipolar myosin II filament can walk towards the actin filament's plus ends, generating maximal contractile force, shown as black arrows. The motor filaments also contain hundreds of heads, which are able to continuously generate force on the filaments and maintain attachment as they hydrolyze ATP to produce mechanical work. (b) In a nonmuscle actomyosin network, filaments are distributed in a random geometric fashion throughout the cytoskeleton. The myosin II filaments in a nonmuscle actomyosin system are also smaller (number of heads per side of bipolar filament $N_{t} \approx 30$ [8]) and highly transient (the duty ratio of bound to unbound states of the motor filament $\rho_{m} \ll 1$ ) compared to their muscle counterparts $\left(N_{t} \approx 500, \rho_{m} \approx 1\right)$, but can form disordered arrangements of locally antiparallel actin filaments. Motor filaments of this nature are responsible for stress fiber formation via compression of a fragmented lamellipodial actin mesh.

passive cross-linkers as force transmitters between neighboring contractile clusters [17-23]. Other studies have also investigated the general nonequilibrium dynamics of such cytoskeletal arrangements, outside of the context of the emergence of contractility, and have described fluidized and glassy network behavior $[17,24]$ as well as strain stiffening of the active polymer gel [25].

In light of both recent and older works, the fundamental physical intuition for the emergence of contractility still largely rests on the classical sarcomeric mechanism, where myosin mini-filament heads are bound to two locally antiparallel actin filaments, allowing the energy-consuming unidirectional walking of those heads to generate a contractile shearing motion between the actin filaments [shown in Fig. 1(a)]. Two questions naturally arise with regard to this picture: (1) How do initially isotropic actin networks in three dimensions give rise to these locally antiparallel contractile force dipoles and (2) do passive cross-linkers play an important role only in percolating force chains at larger spatial scales, as previously suggested, or are they also important in generating contractility at the level of a single force dipole? We will address the first question in a future work.

In this work, we address the second question by analyzing an in silico actomyosin force dipole: two antiparallel actin filaments, connected at their farthest ends by springs to boundaries, allowing both cross-linkers and a myosin mini filament to transiently bind and dissociate. Our analytical and numerical results, in qualitative agreement with recent works discussed below [26,27], indicate that passive cross-linkers play a crucial role already at the scale of a single force dipole, giving rise to a funneled energy landscape, where greater overlap of actin filaments corresponds to a larger number of bound cross-linkers. These configurations have lower free energy compared with less contracted states such that the dipole is energetically biased towards contractile configurations. In addition to shedding light on this gradient of free energy due to cross-linker binding, we have also discovered strongly nonlinear behavior of contractility with respect to the strength of cross-linker binding energy, where a rise of contractile forces is followed by a rapid kinetic arrest of intermediate configurations by strongly bound cross-linkers.

Overall, we found that the thermodynamic drive to more complete overlap of actin filaments is significantly attenuated by trapping in a purely passive force dipole (PFD), generating contractile forces that are noticeably smaller than the ones generated by the unidirectional walking of myosin heads when in the presence of springs counteracting the contraction. However, pure myosin action is also relatively ineffective at generating contractile forces because the bound motor filaments are highly transient, easily permitting recoiling slippage of the contracted element of two oppositely polar actin filaments. We have discovered that crosslinkers can help to overcome this slippage process via a ratcheting mechanism - by using dynamic cross-linkers to prevent recoiling of intermediate configurations without bound motors, amplified contractile forces are produced. This presence of active motor behavior can thus create sharply amplified contractile forces depending on the mechanical and dimensionality constraints of the dipole considered. Our work reveals strong synergy between passive cross-linker binding dynamics and active myosin processes in an active force dipole (AFD), which constitutes the main building block of actomyosin contractile network.

\section{MODELING A FORCE DIPOLE}

To shed light on the thermodynamic and kinetic nature of a fundamental contractile actomyosin element interacting with passive cross-linkers, which serve as building blocks of more complex actomyosin networks in nonmuscle cells, 
we constructed and carried out various simulations of a pair of rigid actin filaments in one dimension (Fig. 2), where cross-linker proteins dynamically bind and unbind to the overlapping region. We first considered a system where only cross-linker binding generates contractile forces in a passive force dipole, followed by simulations where a unidirectional motor was added to form an active force dipole, in which the motor filament can walk in the direction of the plus end of the actin filaments to generate additional mechanical force. We first note that all parameters are given in Tables I and II contained in the Appendixes. Two actin filaments, denoted as the right and left filaments with respect to the simulated length, have length $L=2 \mu \mathrm{m}$ and midpoints $x_{r}$ and $x_{l}$, with initial midpoint positions $x_{r}^{0}=1 \mu \mathrm{m}$ and $x_{l}^{0}=3 \mu \mathrm{m}$, respectively. An overlap length $l_{o}$ between the two filaments can then be defined as $l_{o}=\left(x_{l}+L / 2\right)-\left(x_{r}-L / 2\right)$. Each filament is connected to springs at their outward-facing plus ends such that the tension provided by the springs on each filament is $F_{t}^{r}=$ $-K_{t}\left(x_{r}-x_{r}^{0}\right)$ and $F_{t}^{l}=-K_{t}\left(x_{l}-x_{l}^{0}\right)$. The diversity of the chemical states of the force dipole can be represented with the integer values $(m, n)$, with $m \in[0,1]$ and $n \in[0, \infty]$, which specify the number of (active) myosin II motor filaments and (passive) cross-linkers bound to the filament pair, respectively.

When there are no motors or cross-linkers bound to the pair of filaments $(m=0, n=0)$, they can separately undergo overdamped Langevin motion that includes forces provided by their respective tethers and a stochastic force, via $\eta\left(d x_{l} / d t\right)=F_{t}^{l}+F_{s}$ and $\eta\left(d x_{r} / d t\right)=F_{t}^{r}+F_{s}$, where

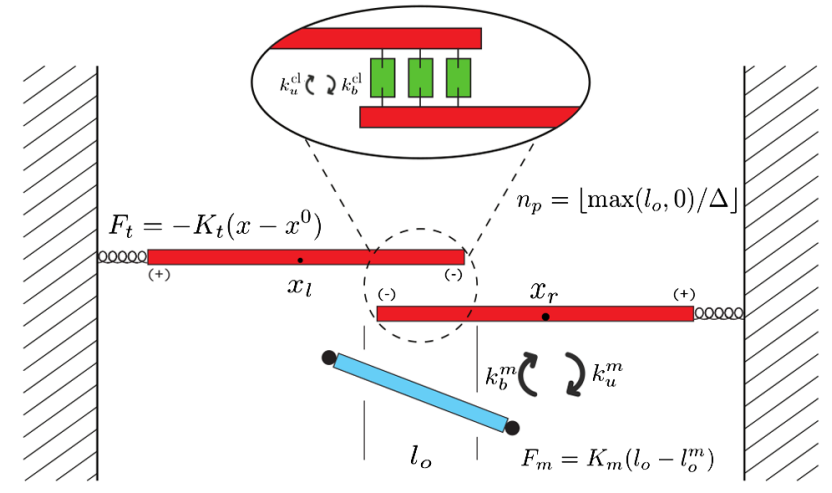

FIG. 2. One-dimensional "force dipole" actomyosin model schematic. Two filaments with plus ends facing outward and midpoints $x_{r}$ and $x_{l}$ are both connected to springs with stiffness $K_{t}$, initially with an equilibrium filament position of $x_{r}^{0}$ and $x_{l}^{0}$. When no cross-linkers are bound between the two filaments, they separately undergo tethered Langevin motion. While the filament overlap $l_{o}$ is large enough, cross-linkers can transiently bind and unbind according to their kinetic rates as in Eq. (2), which arrests the filaments if the number of cross-linkers bound is nonzero.The number of available binding sites $n_{p}$ varies with actin filament overlap. Motor filaments can (un)bind and walk stochastically on the pair of actin filaments, generating force via a time-varying filament overlap potential.
$F_{s}$ satisfies the fluctuation-dissipation theorem in one dimension. The instantaneous number of sites $n_{p}$ available for binding in the overlapping region between the two filaments is obtained from $n_{p}=\left\lfloor\max \left(l_{o}, 0\right) / \Delta\right\rfloor$, where $\Delta$ denotes the spacing between adjacent binding sites. We assume that the filaments move only in the absence of bound cross-linkers $(n=0)$, otherwise, they are immobilized $(n \neq 0)$. We further expect that upon binding of a cross-linker, the tension in each respective actin filament tether is nearly instantaneously equilibrated such that $\left|F_{t}\right| \equiv\left|F_{t}^{r}\right|=\left|F_{t}^{l}\right|$ while preserving the average tensile force experienced by the filament pair at the time step before equilibration, $\left|F_{t}\right|=\frac{1}{2}\left(\left|F_{t}^{l}\right|+\left|F_{t}^{r}\right|\right)$. This equilibration assumption is based on the separation of time scales between tether relaxation ( $\approx \mathrm{ms})$ and (un)binding dynamics $(\approx 0.1 \mathrm{~s}$ in the fastest simulated case). The spatial positions of the filament pair remain stationary until complete crosslinker unbinding $(n=0)$.

The filament pair can also experience an active motor contractile force $F_{m}$, when a motor is bound $(m=1)$, via a stochastically time-varying filament overlap potential, $F_{m}(t)=K_{m}\left[l_{o}(t)-l_{o}^{m}(t)\right]$, where $l_{o}^{m}(t)$ indicates the filament overlap corresponding to an unstretched motor. Upon a new binding event, this motor parameter, which implicitly represents the intrinsic length of the molecule, is initialized to $l_{o}(t)$. This defines the motor filament's equilibrium length in terms of the filament pair's configuration at the moment of the motor's binding. Then, $l_{o}^{m}(t)$ can stochastically increase by a motor step size $d_{s}$ with average walking velocity $v_{w}$, where this velocity depends on the instantaneous value of $F_{m}$, as described in Appendix A. With this active force contribution, the aggregate force experienced by all cross-linkers bound between the filament pair can be written as $F_{\mathrm{cl}}=\left|F_{m}-F_{t}\right|$. The simulation protocol iteratively performs Gillespie stochastic simulation for chemical dynamics (including binding, unbinding, and motorstepping events) and switches to overdamped (mechanical) Langevin dynamics when the number of bound motors and cross-linkers becomes zero (i.e., $m=0$ and $n=0$ ). The latter dynamics is then evolved for $\tau_{r}$, which is the reaction time for the next rebinding event, estimated stochastically based on the same Gillespie algorithm.

Next, we relate the stochastic (un)binding rates of crosslinkers on the filament pair to their thermodynamic binding energy, denoted as $\epsilon$. This binding energy can be phenomenologically related to the change in Helmholtz free energy $\Delta A$ by the following form, which contains enthalpic and entropic contributions [28]:

$$
\Delta A \approx-\epsilon+k_{b} T \ln \left(\frac{v_{m}}{v_{b}}\right)
$$

where $v_{m}=V / N$ is the inverse concentration of $N$ cross-linkers in volume $V$ in solution. $v_{b}$ can be thought of as the binding site volume of the cross-linker, which at most physiological concentrations is much smaller than $v_{m}$. Intuitively from this equation, one can imagine 
system entropy is lowered from cross-linker binding as $k_{b} T \ln \left(v_{m} / v_{b}\right)$, but is counteracted by binding favorability $\epsilon$.

We assume upon cross-linker binding $P \Delta V \approx 0$ such that we can equate the change in Helmholtz free energy $\Delta A$ to the change in Gibbs free energy $\Delta G$. Combining Eq. (1) with this approximation, and using the classic relation for the dissociation of a molecule from a binding site $K_{D}=e^{\Delta G / k_{b} T}$, one obtains an approximate expression for the dissociation constant $K_{D}$ of cross-linkers in this system in terms of their binding energy as

$$
K_{D} \approx \frac{v_{m}}{v_{b}} e^{-\epsilon / k_{b} T}
$$

$K_{D}$ can then be related to the stochastic cross-linker reaction constants by $K_{D}=k_{u}^{\mathrm{cl}} / k_{b}^{\mathrm{cl}}$. Therefore, the concentration of cross-linkers $\left([L] \propto 1 / v_{m}\right)$ as well as cross-linker bond energy $\epsilon$ influences the binding of cross-linkers to the filament pair in this description, with linear and exponential dependencies, respectively. In simulation, we demonstrate the effects of varying the thermodynamic parameter $\epsilon$ for sets of fixed $k_{u}^{\mathrm{cl}}$. This results in variation in $k_{b}^{\mathrm{cl}}$, altering the stochastic dynamics of the force dipole.

Nonmuscle myosin II molecules in vivo are assembled into bipolar mini filaments, with patches of motor heads radiating outward [29] which then can (un)bind to neighboring filament segments. Hence, we must consider motor filament kinetics in our model as a coarse-grained version of a detailed stochastic process including $N_{t}$ transient motor heads per side of the mini filament, coexisting in a connected bipolar structure. The number of motor heads per side of a mini filament is typically $N_{t} \approx 30$ (in total $\approx 60$ heads per mini filament) [8], so it is reasonable to assume that $<10$ of these heads per side could be bound to a single actin filament simultaneously-we choose $N_{t}=10$ for our simulations, which produces a mean unbinding time of $\bar{\tau}_{u}^{m} \approx 1 / \mathrm{s}$ in the absence of mechanosensitivity. Since tension is released upon unbinding of either side of the bipolar filament, we use this value as the effective unbinding rate of our coarsegrained description. Therefore, even with multiple motor filament heads possibly attached to a single actin filament, the stochastic dynamics of these heads creates a highly transient system of tension build and release between the filament pair. We leave the description of our mechanochemical model for these myosin II filaments, which explicitly includes catch-bond dynamics, to Appendix A.

\section{PASSIVE FORCE DIPOLES IN 1D}

In the absence of motor filaments, cross-linkers can create a substantial amount of contractile force in the onedimensional antiparallel filament pair against elastic tethering simply by rectifying Brownian fluctuations which increase filament overlap. Contraction of the PFD can be quantified in terms of a thermodynamic parameter $\epsilon$ and kinetic parameter $k_{u}^{\mathrm{cl}}$ using their previously defined relation. Figure 3(a) shows the average force generated by the dipole $\left\langle F_{D}\right\rangle=\left\langle\left|F_{t}^{l}\right|+\left|F_{t}^{r}\right|\right\rangle$ in 250 simulations for $200 \mathrm{~s}$-we denote this time as $\tau_{\mathrm{lab}}$. We observe a sharply biphasic dependence of generated force on varying crosslinker binding energies. At low $\epsilon$, cross-linkers cannot sustain significant levels of tension between the filaments due to a low occupancy of the available binding sites, while at high $\epsilon$, significant kinetic arrest occurs due to quick
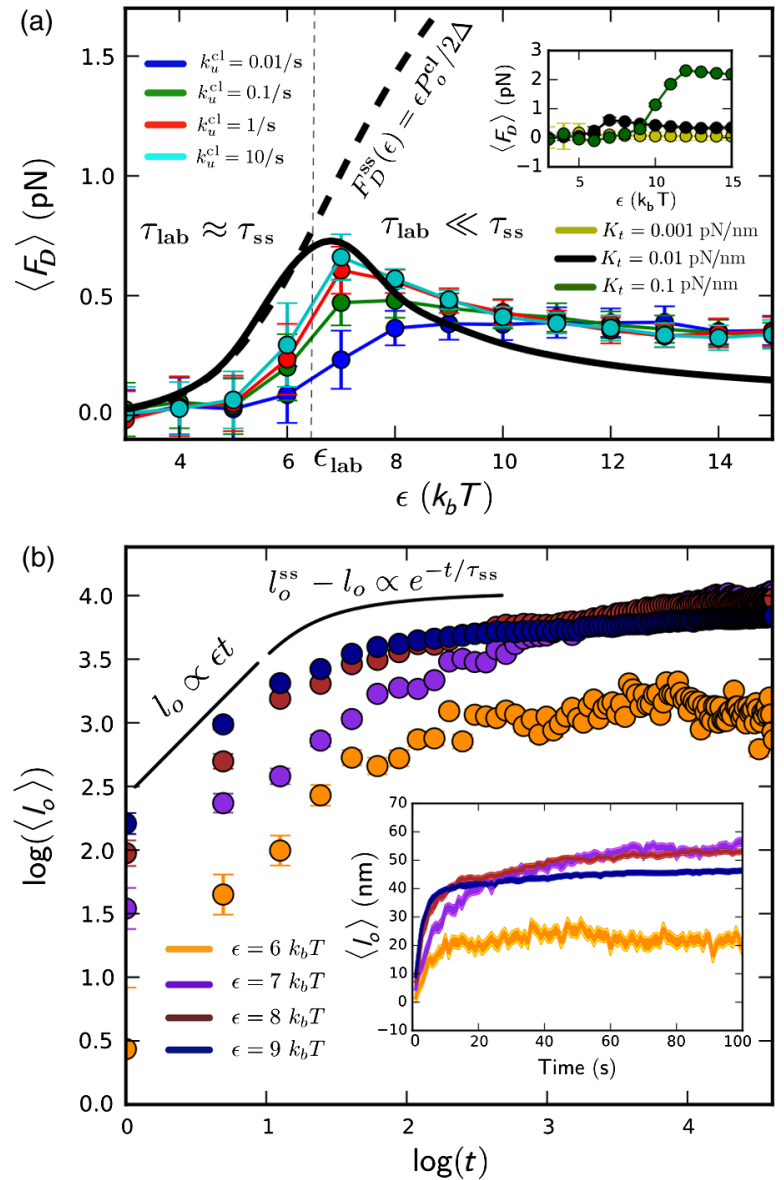

FIG. 3. Cross-linker driven contraction of the passive force dipole in one dimension. (a) Average force $\left\langle F_{D}\right\rangle$ at $\tau_{\text {lab }}=200 \mathrm{~s}$ for $K_{t}=0.01 \mathrm{pN} / \mathrm{nm}$, as a function of cross-linker binding energy $\epsilon$. The analytically predicted $F_{D}^{\text {ss }}$ according to the thermodynamic limit in Eq. (7) is shown as the black dotted curve, with the numerical solution to Eq. (6) as solid black; these solutions diverge at $\epsilon_{\text {lab }} \approx 6 k_{b} T$. This corresponds to the transition $\tau_{\text {lab }} \ll \tau_{\text {ss }}$ caused by increasing binding strength of crosslinks. Increasing speed of unbinding shifts curves to the predicted thermodynamic limit. The inset displays stochastic trajectories for the same cross-linker binding energies. (b) Log-log plot of the average filament overlap $\left\langle l_{o}\right\rangle$ vs time for $\epsilon$ values around $\epsilon_{\text {lab }}$ shows a linear force-generating regime $l_{o} \propto \epsilon t /\left(1+v e^{-\epsilon / k_{b} T}\right)$ followed by an exponential relaxation to steady state: $l_{o}^{\mathrm{ss}}-l_{o} \propto e^{-t / \tau_{\mathrm{ss}}}$, with $\tau_{\mathrm{ss}} \propto\left(1+v^{-1} e^{\epsilon / k_{b} T}\right)^{l_{o} / \Delta}$. Trajectories above $\epsilon_{\text {lab }}$ are far from equilibrium at $\tau_{\text {lab }}=200 \mathrm{~s}$, as shown by a significant flattening of the $l_{o}$ vs $t$ curve at long times for increasing binding energy. 
saturation of the binding sites, significantly hindering filament motion. While cross-linkers are able to generate overlap between the filament pair when restoring forces are minimal, at most binding energies simulated, they are ineffective against stiffer springs counteracting the contraction of the pair $\left(K_{t}=0.1 \mathrm{pN} / \mathrm{nm}\right)$. This is due to slippage of the filament pair when cross-linker dissociation occurs, releasing contractile tension between them. Contraction can only be generated in this case if the binding affinity of the cross-linkers is greater than $10 k_{b} T$, which induces complete arrest of the filament pair upon a single-site occupancy.

Essential to the characteristic force production of the PFD is not only the overall cross-linker affinity $\epsilon$, which controls the probability of occupancy of the $n_{p}$ sites between the filament pair, but also, crucially, the kinetic rates of binding and dissociation processes that determine how quickly initially nonequilibrium dynamics approaches the steady state. The former arise purely from statistical mechanics principles, as we show below. Accelerating cross-linker binding kinetics in this regard [i.e., simultaneously increasing $k_{b}^{\mathrm{cl}}$ and $k_{u}^{\mathrm{cl}}$ while maintaining binding energy as in Eq. (2)] shifts resulting force curves upward until saturation is reached for $k_{u}^{\mathrm{cl}}=10 \mathrm{~s}^{-1}$. This can be thought of as approaching the thermodynamic limit of force production of the dipole as $k_{u}^{\mathrm{cl}} \rightarrow \infty$.

Furthermore, in order to derive a mean-field equation of motion describing the change in filament overlap over time, it would also be useful to have an analytical expression for the mean-first-passage time of unbinding of $n$ cross-linkers from $n_{p}$ cross-link binding sites on the filament pair $\bar{\tau}_{u}^{\mathrm{cl}}$, since this mean behavior defines the rate of filament overlap increase, and thus the time scale of relaxation $\tau_{\mathrm{ss}}$ of the contractile element. In our stochastic representation, this is the mean time of transitioning from the $n=1$ to $n=0$ state. Surprisingly, this mean-passage-time problem is similar to the stochastic dynamics of an ensemble of myosin II motor heads becoming completely unbound from an actin filament [see Appendix A, Eq. (A1)]. Recasting this equation to apply to cross-linker dynamics by replacing the number of motor heads $N_{t}$ with the number of possible binding sites $n_{p}$, as well as cross-linker kinetics of (un) binding, we obtain an expression for the mean passage time of complete cross-linker unbinding:

$$
\bar{\tau}_{u}^{\mathrm{cl}} \approx \frac{1}{k_{b}^{\mathrm{cl}} n_{p}}\left[\left(1+\frac{k_{b}^{\mathrm{cl}}}{k_{u}^{\mathrm{c}}}\right)^{n_{p}}-1\right] .
$$

On the other hand, the mean binding time (i.e., transition time from $n=0$ to $n=1$ ) is simply $\bar{\tau}_{b}^{\mathrm{cl}}=k_{b}^{\mathrm{cl}} n_{p}$. We note that both passage times are fundamentally dependent on the filament overlap by the definition of $n_{p}$ given above. Predictions of Eq. (3) show excellent agreement with simulated first-passage times of cross-linkers from $n=1$ to $n=0$ at various binding site availabilities (data not shown).

With this, we can construct an equation of motion describing the observed stochastic contraction process in terms of the cross-linker's mean-field energetic contribution to the filament pair and the resulting elastic restoring forces of the filament pair. First, in the absence of stochastic effects, a thermodynamic driving overlap force $\epsilon P_{o}^{\mathrm{cl}} / \Delta$ is expected to result when the filament pair increases their overlap by a distance $\Delta$, with a probability of occupancy of the binding site $P_{o}^{\mathrm{cl}}=k_{b}^{\mathrm{cl}} /\left(k_{b}^{\mathrm{cl}}+k_{u}^{\mathrm{cl}}\right)$. Since the motion of the filament pair is hindered if $n \neq 0$, an effective mean-field equation of motion neglecting stochastic force can be written as

$$
2 \eta \frac{d l_{o}}{d t}=\left(\frac{\epsilon}{\Delta} P_{o}^{\mathrm{cl}}-2 K_{t} l_{o}\right)\left(1-\delta_{n}\right),
$$

where

$$
\delta_{n}=\left\{\begin{array}{lll}
0 & \text { if } & n=0 \\
1 & \text { if } & n \neq 0
\end{array} .\right.
$$

Note in this description that our equation contains a random state variable $\delta_{n}$ such that motion is allowed when there are no cross-linkers bound to the filament pair, and $\eta=$ $10^{-3} \mathrm{pNs} / \mathrm{nm}$ is the viscous damping constant of an individual actin filament. Averaged over many intervals of $n=0$ and $n \neq 0$ states, which is valid under the assumption that many intervals occur in motion to a new cross-link position $l_{o} \pm \Delta$, i.e., $\eta \Delta^{2} / \epsilon \gg \bar{\tau}_{b}^{\mathrm{cl}}+\bar{\tau}_{u}^{\mathrm{cl}}$, the $\left(1-\delta_{n}\right)$ term can be separated from the average and evaluated explicitly as the probability of the $n=0$ state during simulation to provide a full mean-field solution. This assumption bounds the kinetic rates considered to $\bar{\tau}_{u}^{\mathrm{cl}} \lesssim 1 \mathrm{~s}$. We express this probability in terms of the cross-linker binding energy as $P\left(n=0 \mid l_{o}\right)=$ $\bar{\tau}_{b}^{\mathrm{cl}} /\left(\bar{\tau}_{b}^{\mathrm{cl}}+\bar{\tau}_{u}^{\mathrm{cl}}\right)=\left[1+v^{-1} \exp \left(\epsilon / k_{b} T\right)\right]^{-l_{o} / \Delta}$, with $v=v_{m} / v_{b}$. This bears similar resemblance in its form to the statistical mechanics problem of Langmuir absorption. We now have a final equation of motion, where we change variables $\tilde{l}_{o}$ to indicate the interval-averaged overlap:

$$
2 \eta \frac{d \tilde{l}_{o}}{d t}=\left(\frac{\epsilon}{\Delta} P_{o}^{\mathrm{cl}}-2 K_{t} \tilde{l}_{o}\right)\left(1+v^{-1} e^{\epsilon / k_{b} T}\right)^{-\tilde{l}_{o} / \Delta} .
$$

This equation predicts a near-linear contractile regime in the short-time limit with a constant velocity: $l_{o} \propto V_{o}^{\mathrm{cl}} t$. This is followed by an exponentially decaying relaxation to steady state: $l_{o}^{\mathrm{ss}}-l_{o} \propto e^{-t / \tau_{\mathrm{ss}}}$ (details of the asymptotic analysis are given in Appendix B). In general, $V_{o}^{\mathrm{cl}}$ is directly proportional to the bond energy and occupancy of cross-linkers in the absence of nonequilibrium effects, and $\tau_{\mathrm{ss}}$ is inversely proportional to the occupancy of the available binding sites. This is observed in our simulations with predicted $V_{o}^{\mathrm{cl}} \propto$ $\epsilon /\left(1+v e^{-\epsilon / k_{b} T}\right)$ and $\tau_{\mathrm{ss}} \propto\left(1+v^{-1} e^{\epsilon / k_{b} T}\right)^{l_{o} / \Delta}$ asymptotic behaviors, as shown in Fig. 3(b). At infinitely long time, the equation of motion predicts the steady-state overlap, 


$$
l_{o}^{\text {ss }}(\epsilon)=\frac{1}{2 \Delta K_{t}} \frac{\epsilon}{1+v e^{-\epsilon / k_{b} T}}
$$

which is shown in Fig. 3(a) as the upper bound of the finitetime solutions which contain kinetic arrest. This steadystate prediction diverges from measurements during the laboratory time scale (i.e., $\tau_{\mathrm{ss}} \gg \tau_{\mathrm{lab}}$ ) when $\epsilon$ passes a threshold of $6 k_{b} T$, which we denote as $\epsilon_{\text {lab }}$. Therefore, our results indicate the sharp onset of a glasslike behavior which produces a transition to far-from-equilibrium filament states. This greatly limits force production of the filament pair when cross-linker affinity becomes greater than $\epsilon_{\mathrm{lab}}$. It is important to note that increasing the crosslink concentration in solution $[L]$ would linearly increase the stochastic rate constant of cross-link binding [Eq. (2)], thus altering the onset of contractile behavior described in Eq. (6).

This analysis shows that a passively cross-linked filament dipole can produce low $\mathrm{pN}$ scale forces, in agreement with recent works [26,27,30,31], only if the cross-links are sufficiently favored to bind to the filament pair from the surrounding solution, forming a funneled free-energy landscape with respect to filament overlap in the force dipole. Despite the manifest nonequilibrium nature of this contraction process, the stochastic trajectories of the dipole

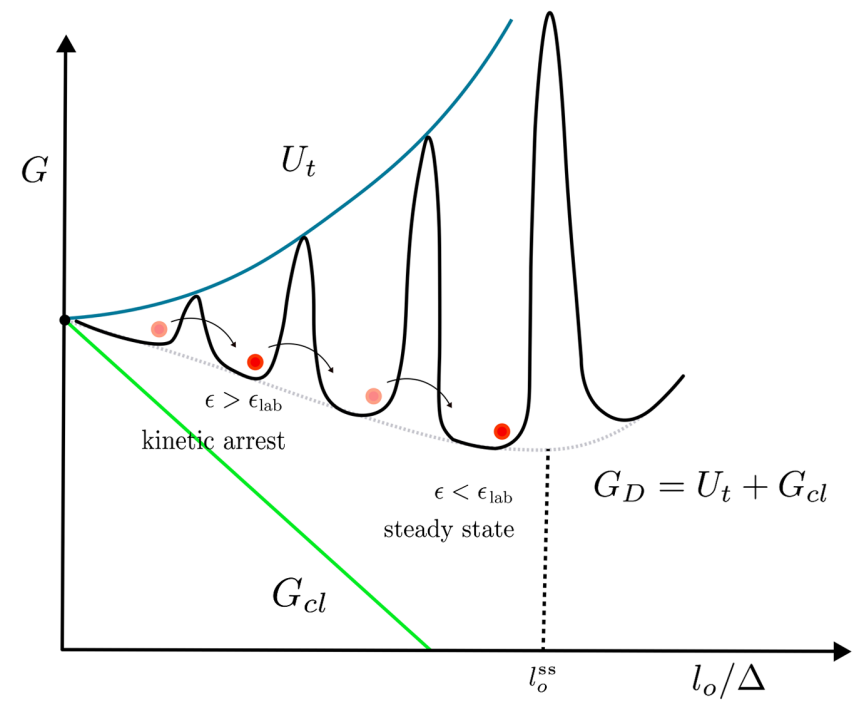

FIG. 4. The free-energy landscape that results from cross-linker binding in a force dipole. The total free energy $G_{D}$ is a summation of cross-link $\left(G_{\mathrm{cl}}\right)$ and tether $\left(U_{t}\right)$ contributions. $G_{\mathrm{cl}}$ is a linear function of overlap, and the slope of this line can be thought of as the average free-energy difference per addition of a single binding site in the dipole (since the number of binding sites $n_{p} \propto l_{o} / \Delta$ ). The dipole's time scale of approach to steady-state overlap (dipole overlap shown as red dots in $G-l_{o} / \Delta$ diagram) is in general an exponential function of cross-link binding affinity such that a dipole can become kinetically arrested on the laboratory time scale if $\epsilon>\epsilon_{\text {lab }}$, and thus $\tau_{\text {lab }} \ll \tau_{\mathrm{ss}}$. Otherwise, the dipole can reach the steady-state free-energy minimum overlap $l_{o}^{\text {ss }}$. could be well understood by treating cross-linkers as exerting a mean-field mechanical driving force of thermodynamic origin. Furthermore, our analysis predicts that this driving force acts to not only favor but also eventually arrest filament overlap depending on the nature of crosslinker binding. A graphical description of this energy landscape with kinetic restrictions is given in Fig. 4.

\section{ACTIVE FORCE DIPOLES IN 1D}

We also show a positive feedback mechanism which produces a force amplification of the one-dimensional cross-linked filament pair when in the presence of active fluctuations. As highly transient myosin II motor filaments are added (the mechanochemical model for motor filaments is outlined in Appendix A) to form an active force dipole in the absence of cross-linkers, the frequent unbinding of the motor filament causes a continual build and release of contractile tension over the simulation duration, resulting in minimal force generation $\left(\approx 0.3 \mathrm{pN}\right.$ against $K_{t}=$ $0.01 \mathrm{pN} / \mathrm{nm}$ springs). However, when cross-linkers are present, three- to 17-fold amplifications are observed compared to the original forces generated in the absence of the motor filament (Fig. 5) at an $\epsilon_{\mathrm{lab}} \approx 5 k_{b} T$. This is shifted from the PFD case, mainly due to myosin II now counteracting restoring tension in the boundary springs, reducing overall tension on cross-linkers in the overlap region and decreasing their effective unbinding rate, consistent with slip-bond feedback (data not shown). We also observe an overall sharp increase in transient motor filament force production when increasing stiffness of external springs, consistent with the myosin II's catch-bond nature $[32,33]$.

At higher external stiffness $K_{t}=0.1 \mathrm{pN} / \mathrm{nm}$, motor filaments alone are more effective than cross-linkers at generating contractile forces for a range of binding affinities $\left(\epsilon \approx 0-10 k_{b} T\right)$ but are still minimally contractile compared to when both passive and active elements are present, which produces $22 \mathrm{pN}$ of force at peak binding affinity. The collective behavior of the one-dimensional actomyosin-cross-linker system is shown in Figs. 5(b) and 5(c) - a "ratcheting" behavior in which the motor, although becoming (un)bound frequently, can steadily produce force over the entire simulation interval, helped by cross-linkers transiently stabilizing filament overlaps. A graphical summary of this behavior is shown in Fig. 5(d).

It is clear that cross-linker trapping of contractile configurations drastically affects the dynamics of an actomyosin dipole. To investigate this effect, we defined in our subsequent analysis two random variables (explicitly dependent on initial filament overlap) which both quantify a change in overlap between filament pairs in a single ratcheting cycle: a contraction function $\chi\left(l_{o}\right)$, in which $(n=0, m=1)$, and an extension function $\xi\left(l_{o}\right)$, in which $(n=0, m=0)$. Both events terminate when $n \neq 0$ and have distance units. Then, by collecting the observed 
(a)

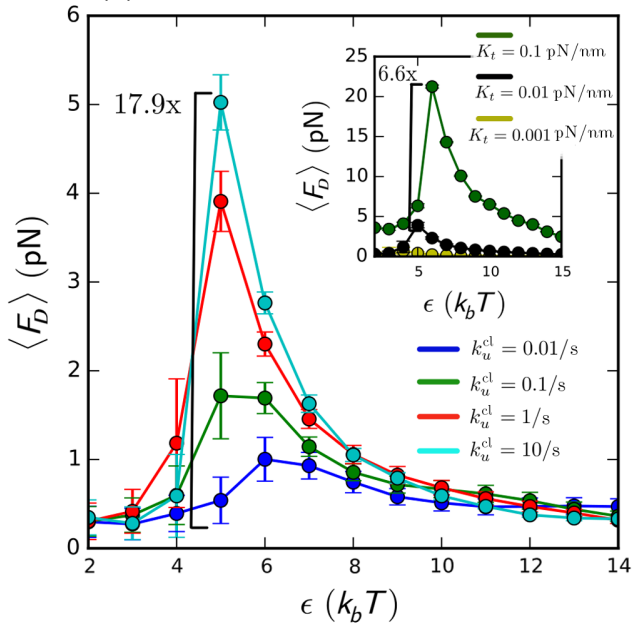

(d) (b)
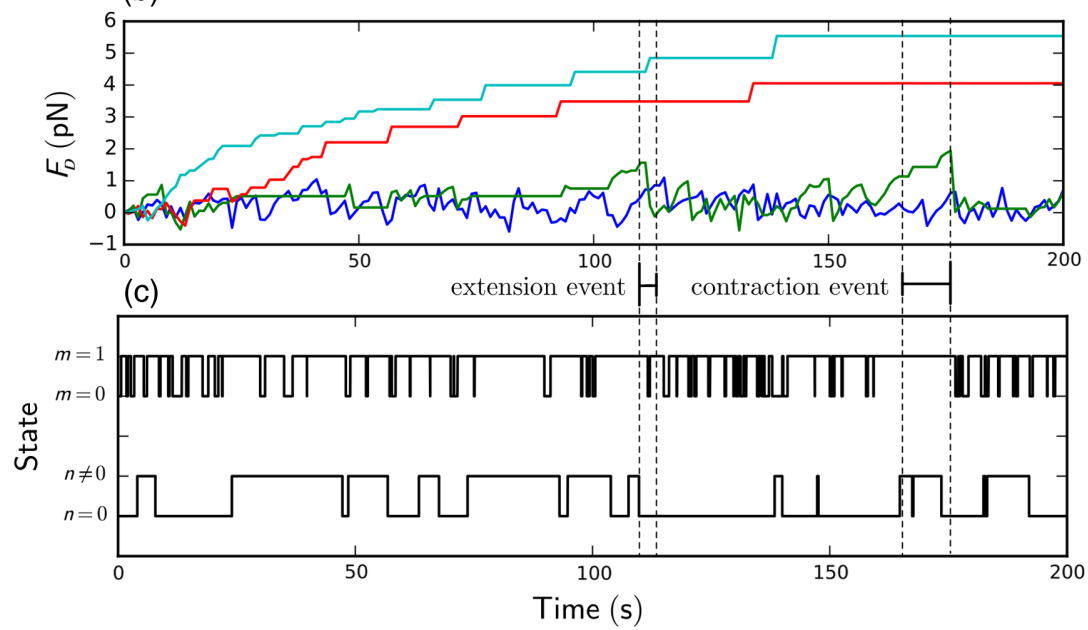
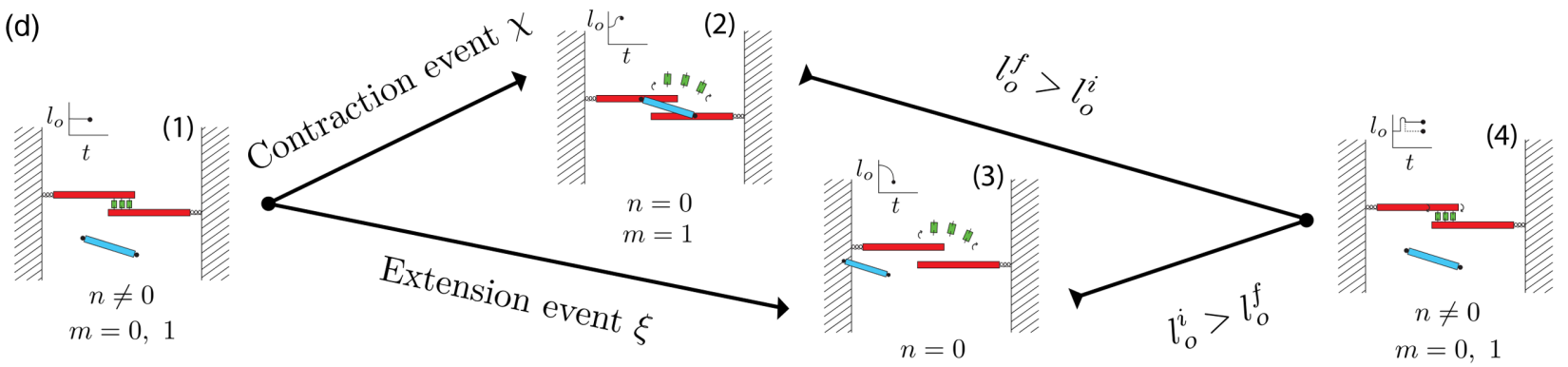

$m=0$

FIG. 5. Actomyosin ratcheting due to transient cross-linking in the one-dimensional active force dipole. (a) Variation of average dipole force $\left\langle F_{D}\right\rangle$ produced on $\tau_{\text {lab }}=200 \mathrm{~s}$ shows similar biphasic behavior compared to a PFD, but is amplified greatly due to the presence of the stochastic motor against a $0.01 \mathrm{pN} / \mathrm{nm}$ tether. Amplifications from the transient motor force are shown $(\approx 0.3 \mathrm{pN})$. Inset shows the effect of varying external stiffness of the diplole $K_{t}$ for a fixed $k_{u}^{\mathrm{cl}}=1 / \mathrm{s}$. The catch-bond nature of myosin II is apparent in the overall increase in generated force at high stiffness. (b) Stochastic force production of the dipole at $\epsilon=5 k_{b} T$ shows a steplike approach to steady state. Increasing kinetic rates maximize overlap generated on $\tau_{\mathrm{lab}}$. (c) The corresponding state trajectory of the same force dipole with $k_{u}^{\mathrm{cl}}=0.1 / \mathrm{s}$, which correspond to contraction and extension events in (b). $m=0,1$ represents the unbound and bound states of the motor, respectively. $n=0$ and $n \neq 0$ represent the cross-linker state. (d) The proposed ratcheting process in an active force dipole. (1) The process starts at a stable configuration $n \neq 0$. The motor can be either bound or unbound $(m=0$ or +1$)$. (2) Cross-linkers then unbind from the dipole $(n=0)$. If $m=+1$, a contraction event occurs, contracting the filaments to a new overlap $l_{o}^{f}>l_{o}^{i}$. (3) If $m=0$, an extension event occurs in which the filaments lose overlap such that $l_{o}^{f}<l_{o}^{i}$. (4) Cross-linkers rebind $(n \neq 0)$, stabilizing the $l_{o}^{f}$ achieved in (1)-(3) in a contracted or extended configuration. This process repeats as pairwise overlap is created between the antiparallel pair, generating contractile force.

overlap change in simulations for each ratcheting cycle, binned by the dipole's initial overlap, we constructed an effective interfilament velocity profile of the AFD, representing the rate at which overlap increases in the filament pair: $V_{\text {eff }}\left(l_{o}\right)=\chi\left(l_{o}\right) \omega_{\chi}\left(l_{o}\right)-\xi\left(l_{o}\right) \omega_{\xi}\left(l_{o}\right)$, where $\omega_{\chi}\left(l_{o}\right)$ and $\omega_{\xi}\left(l_{o}\right)$ represent the frequencies of the respective stochastic events from simulations. Figure 6 shows the simulation results-as binding energy is raised from $\epsilon<$ $\epsilon_{\text {lab }}$ to $\epsilon \approx \epsilon_{\text {lab }}$, contractile velocities decrease significantly at low filament overlaps but become positive at large overlaps due to structural ratcheting, driving maximal contraction of the force dipole at $\epsilon \approx \epsilon_{\mathrm{lab}}$. In agreement with a transition to kinetic arrest observed previously, in the regime $\epsilon>\epsilon_{\text {lab }}$, contraction events are efficient and extension events minimal, but filament motion is severely hindered at large overlaps. This contraction and extension behavior can be reproduced analytically by averaging over the possible kinetic events of contraction and extension in the element-this is derived in Appendix C.

Therefore, the energy landscape induced by transient cross-linking in a passive force dipole was directly observed in the nonequilibrium AFD in our one-dimensional simulations, although in general the steady state and glassy behavior, unlike the PFD dynamics, is not analytically tractable. This active driving resulted in an amplification of contractile forces produced by the dipole, via the ratcheting of motors on the original funneled energy landscape created by cross-linking. Hence, passive cross-linking can play an essential role in contractility, even at the scale of a force dipole containing a single myosin II mini filament. 


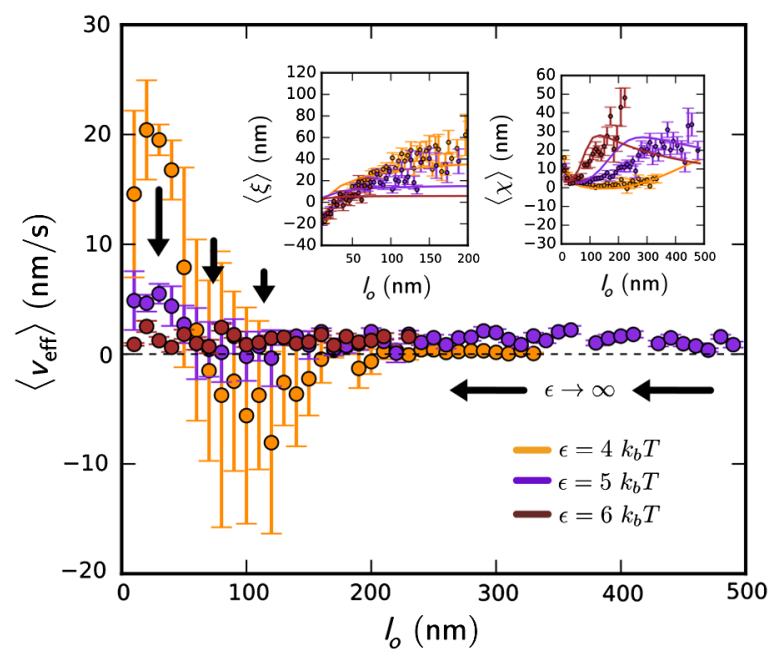

FIG. 6. Effective interfilament velocity in the one-dimensional active force dipole as a function of pairwise overlap, with $k_{u}^{\mathrm{cl}}=$ $10 / \mathrm{s}$ against $K_{t}=0.01 \mathrm{pN} / \mathrm{nm}$ tethers. This plot can be interpreted as the contractile force-velocity relation of the structural element, since pairwise filament overlap $l_{o}$ is directly proportional to the restoring force on filaments due to external tethers. The effective interfilament velocity of the dipole (i.e., the rate at which filament overlap increases) is calculated by combining the change in overlap and frequency of contraction and extension events: $V_{\text {eff }}\left(l_{o}\right)=\chi\left(l_{o}\right) \omega_{\chi}\left(l_{o}\right)-\xi\left(l_{o}\right) \omega_{\xi}\left(l_{o}\right)$. Cross-linking in an optimal range amplifies forces produced by the dipole and is apparent in the effective contractile velocity of the filament pair at large overlaps. $\chi\left(l_{o}\right)$ and $\xi\left(l_{o}\right)$ are also shown in the inset. Analytic approximations for the expected value of these events are showed as filled lines.

\section{ACTIVE FORCE DIPOLES IN 3D}

Until now, we have been considering actin filaments in one-dimensional force dipoles, treating the actin filaments as nondeformable under passive and active motion. This is usually a valid assumption for nonmuscle myosin II mini filaments in an idealized geometry, since motor forces presumably operate under a critical buckling threshold of actin filament segments [34]. Motors interacting with deformable actin filaments and cross-linkers in disordered networks, however, may produce buckling and shearing at the formed cross-linking junctions, providing a channel for active stress dissipation. Therefore, we finally consider the more realistic scenario of semiflexible actin filaments and cross-linkers interacting in three-dimensional geometries.

To investigate the implications of this mechanical feature, we use the recently developed MEDYAN active network model [17], built on previous cytoskeletal modeling efforts in our laboratory [37-40], to generate an in silico threedimensional force dipole. Our setup would correspond to a hypothetical in vitro dual optical trap experiment. More specifically, this model is composed of (1) two semiflexible actin filaments, tethered to springs at their plus ends (to represent optical traps with stiffness $K_{t}$ as will be discussed), coarse grained into cylindrical segments, containing bending and stretching modes and a volume exclusion of pairwise segments, and (2) transient passive cross-linkers and active myosin II motor filaments (both at $1 \mu \mathrm{M}$ ) which can diffuse via a stochastic subvolume reaction-diffusion scheme and (un)bind to neighboring filament segments, coupling them mechanically. A simulation snapshot of this setup is given in Fig. 7. In this simulation protocol, motor filaments can stochastically walk between filament segments when bound, generating active network stress similar to the one-dimensional model. The simulation protocol iteratively performs stochasticreaction diffusion simulation and equilibrates the resulting mechanical deformations due to localized motor motion after a set of chemical events. Details of the simulation protocol are given in Appendix D, with relevant simulation parameters and observables in Table II.

As expected, generated force in the three-dimensional dipole is a strong function of cross-linking affinity, as seen in Fig. 7(b). Overall, 16 independent trajectories of dipoles were run for varying cross-link energy $\epsilon$ and tether stiffness $K_{t}$. An overall amplification of overlap and tether force generation is observed compared to the transient motor forces alone at each stiffness (up to threefold for $K_{t}=0.1 \mathrm{pN} / \mathrm{nm}$ ). Furthermore, we again see a transition to kinetic arrest as evident by a nonlinear decrease in crosslinker fluctuations at all stiffnesses tested [Fig. 7(c)].

On the other hand, these simulations indicate a noticeable transition to a monotonically saturating force at high trap stiffness, unlike the one-dimensional case, which almost entirely decays at large bond energies. We attribute this effect to the ability of cross-linked filaments to rotate from their initial bond position when bound to the antiparallel filaments. Because of cross-linker pivoting, myosin II active motion can then open new possible binding sites by shearing the dipole, without waiting for complete crosslinker unbinding. In particular, extremely high-affinity cross-linkers can arrest those short-lived configurational fluctuations, providing a significant increase in sustained dipole tension. This overall behavior occurs when the characteristic length scale of overlap approaches $l_{o} \approx \Delta$, which corresponds to boundary stiffnesses of $K_{t}=0.1$, $1 \mathrm{pN} / \mathrm{nm}$ in our simulation.

Next, we compare force production observed in our 3D MEDYAN simulations with the corresponding predictions of the 1D model described above. First, let us define the probability of cross-link site occupancy $P_{o}^{\mathrm{cl}}=\left(1+v e^{-\epsilon / k_{b} T}\right)^{-1}$. A power-law relation can be fit by using the 1D steady-state prediction when $\epsilon<\epsilon_{\text {lab }}$, raised to an arbitrary exponent $\alpha$ :

$$
F_{D}^{\mathrm{ss}} \propto\left(\epsilon P_{o}^{\mathrm{cl}}\right)^{\alpha},
$$

where $\alpha$ is obtained by fitting MEDYAN simulation data. In one dimension, $\alpha=1$. The power law is shown in Fig. 7 
(a) $\quad \mathbf{x}_{l}^{t} \mathbf{x}_{l}^{p}$

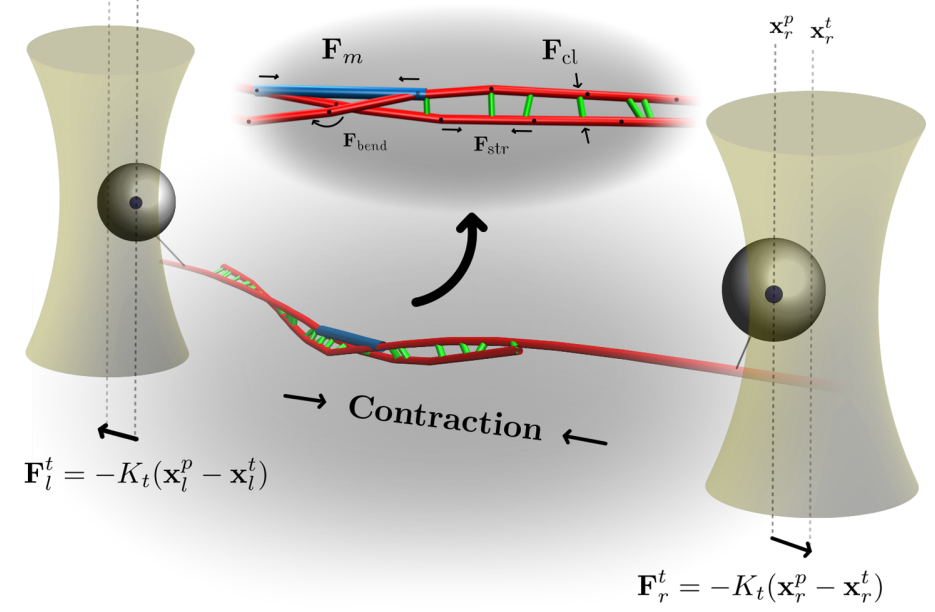

(b)

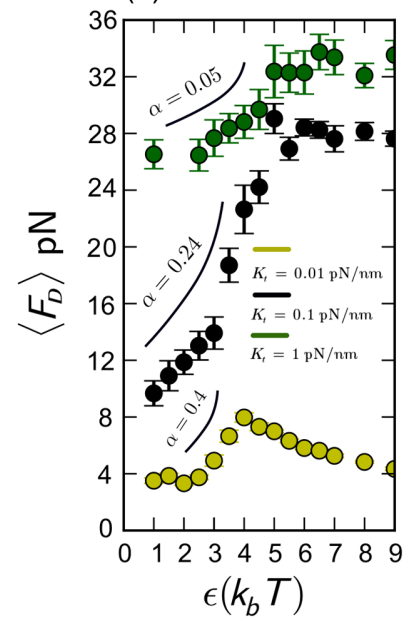

(c)

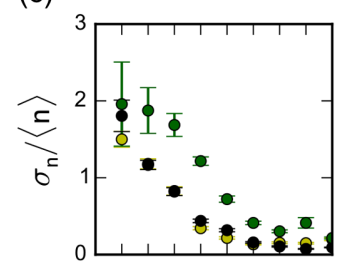

(d)

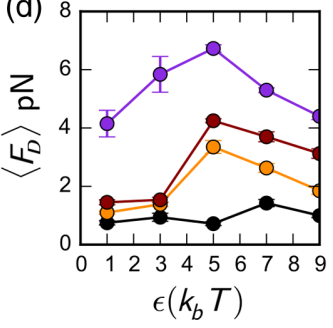

FIG. 7. A three-dimensional force dipole simulated using MEDYAN, an active matter reaction-diffusion model. (a) A schematic of the simulation setup, very similar to the one-dimensional model shown in Fig 2. Two filaments (red), now containing three-dimensional bending and stretching modes, are oriented so that their plus ends $\left[\mathbf{x}_{l}^{p}=(0,0,0) \mu \mathrm{m}\right.$ and $\mathbf{x}_{r}^{p}=(4,0,0) \mu \mathrm{m}$, respectively, at $\left.t=0\right]$ are connected to elastic, stationary tethers at $\mathbf{x}_{l}^{t}$ and $\mathbf{x}_{r}^{t}$ (initially $\mathbf{x}_{l, r}^{p}=\mathbf{x}_{l, r}^{t}$ ). As contraction is produced by myosin II filaments (blue) walking towards the plus ends of each actin filament, cross-linkers (green) and actin filaments can bend and stretch according to polymer potentials outlined in Appendix D. (b) The averaged dipole force after $200 \mathrm{~s},\left\langle F_{D}\right\rangle$, is shown as a function of $\epsilon$. The steady-state data follow the power laws $F_{D}^{\text {ss }} \propto\left(\epsilon P_{o}^{\mathrm{cl}}\right)^{\alpha}$, where $\alpha=0.4,0.24$, and 0.05 , respectively, for increasing $K_{t}$. The probability of a single-site occupancy $P_{o}^{\mathrm{cl}}$ is given by $\left(1+v e^{-\epsilon / k_{b} T}\right)^{-1}$. A diminishing effect of cross-linker ratcheting is explained by myosin II's ability to increase its binding lifetime under stress, allowing for more stable walking and force generation at $K_{t}=1 \mathrm{pN} / \mathrm{nm}$. (c) Fluctuations of bound cross-linkers (defined as $\sigma_{n} /\langle n\rangle=\langle n-\langle n\rangle\rangle /\langle n\rangle$ ) show a sharp onset of kinetic arrest at $\epsilon=\epsilon_{\text {lab }}$, similar to the one-dimensional case. (d) Average dipole force produced for varying initial overlap $l_{o}^{i}=1000,500,200,0 \mathrm{~nm}$, with $l_{o}^{i}=0$ as the top curve. Increasing initial overlap $l_{o}^{i}$ will decrease contractile force produced in simulation $\left(\tau_{\text {lab }}=200 \mathrm{~s}\right)$ due to kinetic friction.

and gives exponents of $\alpha=0.4,0.24$, and 0.05 for external stiffnesses of $K_{t}=0.01,0.1,1 \mathrm{pN} / \mathrm{nm}$, respectively. A decrease in $\alpha$ indicates a transition to a motordominated regime of force production due to the motor's catch-bond nature, as predicted at stiffnesses greater than $1 \mathrm{pN} / \mathrm{nm}$ for myosin IIA [33]. Therefore, dipole contractility is most amplified by cross-linking when external stiffness is below this threshold.

We also tested whether initial overlap of the actin filament pair as well as initial incidence angle (as long as initially within pairwise binding range of myosin II) have intuitive effects on dipole dynamics. If the actin filaments have some initial overlap $l_{o}^{i}$ while still initially in a $\left\langle F_{D}\right\rangle=0$ state (starting at $t=0$ ), the overall tension produced in the dipole on $\tau_{\text {lab }}$ is decreased due to newly available binding sites causing additional kinetic friction as previously described. Still, these simulations display characteristics of the passive cross-linker energy landscape and follow a similar $F_{D}$ vs $\epsilon$ trend. Overall, actomyosin dipoles provide force in an overlap-dependent manner-this is shown in Fig. 7(d). Incidence angle of filaments, in contrast (data not shown), has no effect on their resulting contractile force, since the combination of myosin II and passive cross-linking drives the filaments to tense antiparallel orientations relative to the dual traps, regardless of initial crossing geometry.
In summary, cross-linker deformability in a threedimensional force dipole results in new channels of active stress dissipation, as well as a transition to monotonically saturating forces at high binding energies. Surprisingly, actin filament deformability had no effect on overall dynamics in our analysis_-increasing the bending modulus of actin filaments fourfold caused no changes in force production at all cross-linker binding energies. These data are not shown, for brevity.

\section{DISCUSSION}

In this paper, we have investigated the ability of a pair of actin filaments to contract by rectifying thermal and active motions in stochastic models with varying complexity. The contraction of these elements is essential in understanding the macroscopic dynamics of cross-linked actin gels undergoing myosin II rearrangement in nonmuscle systems, but differs from the classic sarcomeric contractility mechanisms that neglect passive cross-linkers as potential force producers. We found that the free-energy landscape induced by the favorable binding of passive cross-linkers can create noticeable contractile force in a one-dimensional filament pair, however, typically in a biphasic window of binding affinities. This adds a crucial regime of behavior to the continuum description of Walcott and Sun, who 
predicted a monotonic contractile filament sliding with cross-linker bond energy without this arrested state [26].

While passive cross-linkers in the absence of myosin II in our simulations lead to force production within the pair of filaments, these forces in general are smaller than forces generated by myosin II motors. The latter by themselves also become rather inefficient, however, due to overlap slippage caused by restoring forces when myosin II transiently dissociates, which is frequent because of their low processivity at low external stiffness $[32,33]$. Hence, in this work, we have discovered that simultaneous combination of all three components, namely, actin filaments, cross-linkers with a sufficient binding affinity, and myosin II, can produce a highly contractile active force dipole due to a stochastic amplification mechanism where cross-linkers trap contractile states as transient motors (un) bind frequently. Also, importantly, cross-linker deformability must be considered for a realistic representation of actomyosin force dipole dynamics. In particular, myosin II's ability to actively shear the dipole and produce additional binding sites without complete cross-linker dissociation generates a specific mode of contraction which is monotonically dependent on passive cross-linker binding affinity. This should be observed in systems where the characteristic distance between cross-links is on the same order of magnitude as the generated overlap between filaments. Otherwise, cross-linkers' effect on overlap generation should be biphasic.

Our simulations give predictions for a possible optical trapping experiment, similar to Refs. [27,41], but including a dual trap with two actin filaments anchored to separate beads, which can then transiently interact with crosslinking proteins and myosin II motor filaments. This setup would measure the time evolution of trap restoring forces $\left\langle F_{t}^{r}\right\rangle$ and $\left\langle F_{t}^{l}\right\rangle$ upon interacting with motor filaments and cross-linkers. Mainly, we would predict a strong amplification of the original $\left\langle F_{D}\right\rangle$ vs $[L]$ relation (i.e., the passive force dipole force production) when motor filaments are added to the system, producing force greater than the sum of individual motor and cross-linker contributions. We also expect to observe a strongly biphasic behavior of contractile force with respect to variation of concentration $[L]$ or cross-link binding affinity $\epsilon$, with a kinetic arrest regime where cross-linker and filament fluctuations decay exponentially, including shift to monotonic force production at trap stiffnesses greater than $0.1 \mathrm{pN} / \mathrm{nm}$.

While we have chosen to use $\epsilon$ as the main control variable in this paper, our description can also predict behavior with respect to variation in cross-linker concentration $[L]$, mainly that in the dual optical trapping experiment, $F_{D}^{\mathrm{ss}} \propto[L]^{\alpha}$ at steady state, where $\alpha$ is sublinear $(\alpha=0.4,0.24$, and 0.05 in our simulations for $K_{t}=0.01-1 \mathrm{pN} / \mathrm{nm}$ ). Similarly, we also predict an exponential increase in relaxation time scales with increasing overlap and cross-linking affinity, in qualitative agreement with recent experiments by Lansky et al. of exponentially increasing friction with bound cross-linkers in microtubule assays [27].

In summary, we have shown that robust contraction can occur in the fundamental nonmuscle actomyosin building block due to a mechanism arising from the energetic favoring of increased passive cross-linking, independent of mechanical network symmetry breaking (i.e., buckling of filaments as previously shown [14,15]). Overall, we envision a disordered actomyosin network to be composed of highly contractile PFDs and AFDs, where the emergent contractility of an entire network, in general, is not a linear combination of dipolelike contractions in the comprising elementary units. In this case, the relative importance of these elements should be largely determined by boundary conditions and steady-state network microstructures. It will be an interesting continuation of this study to investigate those nonlinear emergent behaviors in more realistic three-dimensional environments using the dipole theory presented.

\section{ACKNOWLEDGMENTS}

We thank Alex Mogilner for helpful discussions during the initial phase of this project. We are also grateful to Aravind Chandrasekaran, Cal Floyd, and Hao Wu for their review of the manuscript. All simulations were performed on the Deepthought2 Supercomputer at the University of Maryland. This work was funded by National Science Foundation Grant No. CHE-1363081 and No. DGE1632976.

\section{APPENDIX A: PARAMETER CONSIDERATIONS FOR THE SIMULATED PROTEINS}

The parameters chosen for our simulations are given in Tables I and II, which includes experimentally measured mechanical stiffnesses as well as chemical kinetics of (un)binding for the proteins considered. Considering cross-linkers in vivo, the abundant cross-linker $\alpha$-actinin displays a bound lifetime of $2.5 \mathrm{~s}$ and $\Delta G$ of $2-4 k_{b} T$ as addressed in recent reviews $[30,42]$. Other abundant cytosolic cross-linker filamin has a lifetime of $\approx 1 \mathrm{~s}$ and similar free energy of binding. Other cross-linkers not found in contractile actomyosin, such as fascin, have a much stronger binding of $\Delta G=15 k_{b} T$. So, we choose to vary a binding energy control variable $\epsilon$ from 0 to $15 k_{b} T$. We also use kinetic (un)binding rates for cross-linkers in the afore-mentioned physiological range while also testing slower kinetics: $k_{u}^{\mathrm{cl}}$ is varied from 0.01 per s to 10 per s.

Although the aim in this study is not to probe the effects of mechanochemical feedback between these proteins, we find it necessary to include key mechanochemical relationships for full model realism. For individual cross-linker bound to the filament pair, we employ a typical "slip" bond characterized by a decreasing bound lifetime with applied load [41] as $k_{u, \text { eff }}^{\mathrm{cl}}=k_{u}^{\mathrm{cl}} e^{F_{\mathrm{cl}} x_{\mathrm{cl}} / n_{p} k_{b} T}$, where $x_{\mathrm{cl}}$ is a characteristic 
TABLE I. Glossary of variables and model parameters chosen to mimic a typical system of actin filaments, cross-linkers, and nonmuscle myosin IIA motor filaments in one dimension.

\begin{tabular}{|c|c|c|}
\hline Parameter & Description & Value \\
\hline$k_{b} T$ & Thermal energy & $4.1 \mathrm{pN} \mathrm{nm}$ \\
\hline$x_{l, r}^{0}$ & Initial left $(l)$ and right $(r)$ actin filament midpoints & $1 \mu \mathrm{m}, 3 \mu \mathrm{m}$ \\
\hline$L$ & Length of actin filament & $2 \mu \mathrm{m}$ \\
\hline$K_{t}$ & Boundary tether stiffness & $0.001-1 \mathrm{pN} / \mathrm{nm}$ \\
\hline$\eta$ & Viscous damping constant & $10^{-3} \mathrm{pNs} / \mathrm{nm}$ \\
\hline$F_{s}$ & Stochastic force experienced by actin filaments & $\cdots$ \\
\hline$l_{o}$ & Actin filament pairwise overlap & Observable \\
\hline$F_{t}^{l, r}$ & Tether forces experienced by left $(l)$ and right $(r)$ actin filaments & Observable \\
\hline$F_{\mathrm{cl}}$ & Force experienced by bound cross-linkers & $\cdots$ \\
\hline$F_{m}$ & Force experienced by motor filament & $\cdots$ \\
\hline$F_{D}$ & Total dipole force (sum of left and right tether tensions) & Observable \\
\hline$x_{l, r}$ & Instantaneous left $(l)$ and right $(r)$ actin filament midpoint & $\cdots$ \\
\hline$l_{o}^{m}$ & Motor-preferred actin filament overlap & $\cdots$ \\
\hline$l_{o}^{\mathrm{ss}}$ & Steady-state overlap in PFD & $\cdots$ \\
\hline$N_{t}$ & Number of single motor heads per side of motor filament & $10^{\mathrm{a}}$ \\
\hline$m, n$ & Number of bound motor filaments $(m)$ and cross-linkers $(n)$ & $\cdots$ \\
\hline$n_{p}$ & Number of possible cross-link binding sites & $\cdots$ \\
\hline$\epsilon$ & Cross-linker binding energy & $0-15 k_{b} T$ \\
\hline$v_{m}$ & Effective volume of cross-linker in solution & $1 \times 10^{-3} \mu \mathrm{m}^{3 \mathrm{~b}}$ \\
\hline$v_{b}$ & Approximate bound volume of cross-linker & $3 \times 10^{-6} \mu \mathrm{m}^{3 \mathrm{c}}$ \\
\hline$\Delta$ & Distance between cross-link binding sites & $10 \mathrm{~nm}$ \\
\hline$k_{b, u}^{\mathrm{cl}, m}$ & (Un)binding rate of cross-linkers $(\mathrm{cl})$ and motor $(\mathrm{m})$ & For $\mathrm{cl}, 0.01-10 / \mathrm{s}$ \\
\hline $\bar{\tau}_{b, u}^{\mathrm{cl}, m}$ & Mean (un)binding time of cross-linkers $(\mathrm{cl})$ and motor $(m)$ & $\cdots$ \\
\hline$d_{s}$ & Motor filament step size & $5 \mathrm{~nm}$ \\
\hline$k_{b, u}^{\mathrm{ms}}$ & Single motor head (un)binding rate & $0.2 / \mathrm{s}, 1.7 / \mathrm{s}[43]$ \\
\hline$F_{s}$ & Motor filament stall force & $24 \mathrm{pN}^{\mathrm{d}}$ \\
\hline$v_{w}^{0}$ & Walking velocity of motor filament & $10 \mathrm{~nm} / \mathrm{s}^{\mathrm{e}}$ \\
\hline$\alpha$ & Stall velocity mechanochemical parameter for motor filament & $0.2[32]$ \\
\hline$\beta$ & Catch-bond mechanochemical paramter for motor filament & $2[32]$ \\
\hline$x_{\mathrm{cl}}$ & Characteristic slip length for cross-linker & $0.5 \mathrm{~nm}[41]$ \\
\hline$x_{m}$ & Characteristic catch length for single motor head & $1.6 \mathrm{~nm}[44]$ \\
\hline$K_{\mathrm{ms}}$ & Stiffness of single motor head light chain & $0.5 \mathrm{pN} / \mathrm{nm}[45,46]$ \\
\hline$K_{m}$ & Effective spring constant of motor filament & $\cdots$ \\
\hline$\tau_{\text {lab }}$ & Time scale of laboratory measurements & $200 \mathrm{~s}$ \\
\hline$\epsilon_{\text {lab }}$ & Divergence point of steady-state and laboratory measurements & $\cdots$ \\
\hline$\chi$ & Stochastic contraction length of AFD & Observable \\
\hline$\xi$ & Stochastic extension length of AFD & Observable \\
\hline$\omega_{\chi}$ & Contraction frequency of AFD & Observable \\
\hline$\omega_{\xi}$ & Extension frequency of AFD & Observable \\
\hline$V_{\text {eff }}$ & Effective interfilament velocity of AFD & Observable \\
\hline
\end{tabular}

${ }^{\mathrm{a}}$ Geometric constraints of the bipolar motor filament may disallow all tens of heads to be available for binding to a pair of actin filaments due to its double-ended conic structure $[8,47]$. Since the number of nonmuscle myosin II heads per side of a mini filament is 30 for isoform A [8], we assume for this study that a third of these heads are available for binding to the 1D actin filament.

${ }^{\mathrm{b}}$ Calculated as the inverse of concentration $V / N$, assuming a bulk cross-linker concentration of $1 \mu \mathrm{M}$.

${ }^{c}$ Although the exact volume of a cross-linker binding pocket is unknown, this is an order of magnitude estimate based on the dimensions of $\alpha$-actinin [48], which is valid in the approximate expression of Eq. (2). Changes of this value would alter the effective stochastic rate constants of cross-linker (un)binding for a given $\epsilon$.

${ }^{\mathrm{d}}$ An approximation using the stall force of a single myosin II head, $F_{\mathrm{ss}} \approx 4 \mathrm{pN}$. In general agreement with [49], multiplied by its duty ratio at stall and the number of motor heads available for binding, $F_{s}=\rho_{s}^{m}(F) N_{t} F_{\mathrm{ss}}=24 \mathrm{pN}$.

${ }^{\mathrm{e}}$ Calculated using the analytic result of Erdmann et al. [32] for the zero-force walking rate based on individual motor head (un)binding rates. This value was also approximately obtained by Stam et al. [33] and is further motivated by the experimental results of Norstrom et al. [47]. 
TABLE II. Model parameters chosen to mimic a typical system of actin filaments, cross-linkers, and nonmuscle myosin IIA motor filaments in three-dimensional MEDYAN simulations of an actomyosin force dipole. All other parameters and models used in the onedimensional stochastic "toy" representation, but not included in this table, are directly implemented as in the previous model. This includes motor and cross-linker mechanosensitivity.

\begin{tabular}{|c|c|c|}
\hline Parameter & Description & Value [for stochastic kinetics] \\
\hline$D_{a}$ & Actin diffusion coefficient & $20 \mu \mathrm{m}^{2} \mathrm{~s}^{-1}\left[80 \mathrm{~s}^{-1}\right]^{\mathrm{a}}$ \\
\hline$D_{\mathrm{cl}}$ & $\alpha$-actinin diffusion coefficient & $D_{a} / 10 \mu \mathrm{M}^{-1} \mathrm{~s}^{-1}$ \\
\hline$D_{m}$ & Motor filament diffusion coefficient & $D_{a} / 100 \mu \mathrm{M}^{-1} \mathrm{~s}^{-1}$ \\
\hline$k_{b, u}^{\mathrm{cl}}$ & Cross-linker unbinding rate & $\epsilon$ dependent, $1.0 \mathrm{~s}^{-1}[43]$ \\
\hline$l_{c}$ & Length of actin filament segment & $108 \mathrm{~nm}$ \\
\hline$l_{p}$ & Actin filament persistence length & $17 \mu \mathrm{m}[50]$ \\
\hline$\epsilon_{\text {bend }}$ & Actin filament bending energy & $672 \mathrm{pN} \mathrm{nm}$ \\
\hline$K_{\text {str }}$ & Actin filament stretching constant & $100 \mathrm{pN} / \mathrm{nm}$ \\
\hline$K_{\mathrm{vol}}$ & Actin filament excluded volume constant & $10^{5} \mathrm{pN} / \mathrm{nm}^{4}$ \\
\hline$N$ & Number of compartments in each dimension & $6,6,6$ \\
\hline$l_{K}$ & Kuramoto (compartment) length & $500 \mathrm{~nm}^{\mathrm{a}}$ \\
\hline$g_{\mathrm{tol}}$ & Gradient minimization tolerance & $0.1 \mathrm{pN}^{\mathrm{a}}$ \\
\hline$t_{\min }$ & Time step between mechanical equilibration & $0.05 \mathrm{~s}^{\mathrm{a}}$ \\
\hline
\end{tabular}

${ }^{\mathrm{a}} \mathrm{An}$ explanation of this parameter is given in the original MEDYAN paper [17].

unbinding distance and $k_{u}^{\mathrm{cl}}$ is the zero-force unbinding rate. We only consider pulling forces such that $F>0$.

We now consider the mechanochemical dynamics of the myosin II motor filament included in our model. These highly transient units, as described in the main text, must be regarded as a coarse-grained version of a more detailed stochastic (un)binding and walking process of many individually transient motor heads in a single bipolar mini filament. We consider an effective unbinding rate of the coarse-grained overlap potential which mimics the mean unbinding time of a single side of the mini filament with $N_{t}=10$ heads. Since the overall motor filament unbinding rate $k_{u}^{m}$ is nontrivial compared to the single cross-linker case because of its nonlinear dependence on the single motor head (un)binding rates $k_{b}^{\mathrm{ms}}$ and $k_{u}^{\mathrm{ms}}$ and individual motor head stochasticity, we use an approximate expression for the mean unbinding time of the ensemble of heads derived by Erdmann et al. [32] using a series expansion of an adjoint master equation for motor ensemble states:

$$
\bar{\tau}_{u}^{m} \approx \frac{1}{k_{b}^{\mathrm{ms}} N_{t}}\left[\left(1+\frac{k_{b}^{\mathrm{ms}}}{k_{u}^{\mathrm{ms}}}\right)^{N_{t}}-1\right] .
$$

We note that this form is accurate only under a zero-load assumption, but is remedied by combining with an exponential factor as shown below. In an approximation, we inherently assume a single exponentially distributed process with rate $k_{u}^{m}=1 / \bar{\tau}_{u}^{m}$. For simplicity, we also consider motor filament binding to the pair of actin filaments as a single stochastic process with rate $k_{b}^{m}=k_{b}^{\mathrm{ms}} N_{t}$, ignoring partially bound states which do not generate tension between the actin filament pair.

Individual myosin II motor heads have been shown to display "catch-bond" behavior, characterized by an increased bound lifetime with applied load [43,44,51]. We aim to describe the (un)binding and walking kinetics of an ensemble of motor heads with a simple set of parameters to capture the essential aspects of motor filament mechanosensitivity - a binding lifetime that increases exponentially with applied load $F$ and the number of motor heads in the filament $N_{t}$, motivated by the results of Ref. [32], and a walking velocity that is one of the celebrated Hill form [5]. Since the exponential dependence of motor head unbinding in the post-power-stroke state has been directly observed in the bound lifetime of an entire ensemble of heads [32], we express the unbinding rate of the motor filament as

$$
k_{u, \mathrm{eff}}^{m}=k_{u}^{m} e\left(\frac{-F x_{m}}{N_{b}(F) k_{b} T}\right),
$$

where $x_{\mathrm{cl}}$ is a characteristic unbinding distance of a single motor head, and $N_{b}(F)$ is the number of motor heads per side of the motor filament bound to actin. An essential characteristic of the myosin II motor filament to capture in this function is a near-linear increase in the number of bound heads with external load $[6,32,52]$. Since this expression is difficult to derive due to the stochastic nature of the motor heads, an approximate expression for $N_{b}(F)$, motivated by Ref. [32], can be written as $N_{b}(F)=\rho N_{t}+\beta F$, where the mechanosensitivity parameter $\beta$ has been chosen to mimic the response of a low-duty ratio motor like nonmuscle myosin IIA. Upon a motor stepping event, the number of bound heads determines the effective spring constant of the bipolar mini filament with $N_{t}$ heads bound per side to an actin filament in parallel, with stiffness $K_{\mathrm{ms}}$ corresponding to the myosin II light chain connecting individual heads. The sides of the bipolar filament are mechanically connected by an extremely stiff motor filament heavy chain 
region, giving an effective spring constant of the ensemble $K_{m}=K_{\mathrm{ms}} N_{t} / 2$.

In the case of motor walking, we can include a forcedependent walking velocity $v_{w}\left(F_{m}\right)$ similar to a Hill relation [5] for myosin II:

$$
v_{w}(F)=2 v_{w}^{0} \frac{F_{s}-F_{m}}{F_{s}+F / \alpha},
$$

where $F_{m}$ is the instantaneous force on the motor, $\alpha$ is a parameter describing the concave nature of the velocity function which has been chosen to mimic a low-duty ratio motor, and the stall force of the motor filament $F_{s}$ is described in Table II. This equation accounts for two ensembles of heads walking with velocity $v_{w}^{0}$ in opposite directions on each actin filament. We note that, in general, nonmuscle myosin II filaments are highly transient compared to their muscle sarcomere counterpart, but this still has shown to be an effective representation of stall dynamics [32]. This transience is due to a small number of motor molecules $N_{t} \approx 28$ [8] as compared to smooth or skeletal muscle filaments where $N_{t} \approx 500$ [52], causing frequent detachments of the motor head ensemble from the filament pair and tension release, which, in turn, raises serious slippage issues, as discussed.

We have chosen in our stochastic representation to model nonmuscle myosin II isoform A, which displays a faster head (un)binding dynamics and less mechanosensitivity compared to isoform B, another abundant motor filament in the eukaryotic cytosol [51]. In in vitro sliding assays, myosin II head sliding velocities vary significantly, ranging from $50 \mathrm{~nm} / \mathrm{s}$ to as slow as $10 \mathrm{~nm} / \mathrm{s}$ under varying ATP concentration for isoform $\mathrm{B}[8,47]$. Although to our knowledge no single-molecule kinetic study of isoform A mini filaments in vivo has been performed, we use a prediction from Erdmann et al. [32] to obtain an average isoform A sliding velocity of $v_{w}^{0}=10 \mathrm{~nm} / \mathrm{s}$ based on the isoform and number of binding heads we are considering in the mini filament:

$$
k_{w}^{m}=\left(\rho_{m s}{ }^{-1}-1\right) k_{b}^{\mathrm{ms}},
$$

where $\rho_{\mathrm{ms}} \approx 0.1$ is the zero-force duty ratio of a single motor head determined experimentally [51].

\section{APPENDIX B: POWER-LAW BEHAVIOR OF THE PASSIVE FORCE DIPOLE}

We here perform a short-time and asymptotic analysis of the contraction equation presented in this paper [Eq. (6)]. We rewrite the original contraction ordinary differential equation as

$$
\frac{d l_{o}}{d t}=a e^{-\gamma l_{o}}-b l_{o} e^{-\gamma l_{o}}
$$

where $\quad a \equiv \epsilon /\left(1+v e^{-\epsilon / k_{b} T}\right) 2 K_{t} \eta, \quad b \equiv K_{t} / \eta, \quad$ and $\quad \gamma \equiv$ $\ln (c) / \Delta$, where $c \equiv 1+v^{-1} e^{\epsilon / k_{b} T}$. We can also write the predicted steady-state overlap as $l_{o}^{\text {ss }} \equiv(b / a)$. This equation is now separable and yields

$$
\operatorname{Ei}\left(-\gamma l_{o}^{\mathrm{ss}}\right)-\operatorname{Ei}\left[\gamma\left(l_{o}-l_{o}^{\mathrm{ss}}\right)\right]=b t e^{-\gamma l_{o}^{\mathrm{ss}}},
$$

where $\operatorname{Ei}(\cdot)$ represents the exponential integral $\operatorname{Ei}(x)=$ $-\int_{-x}^{\infty} e^{-x} d x / x$. We can expand in the short-time limit, i.e., at $l_{o} \rightarrow 0, \operatorname{Ei}\left[\gamma\left(l_{o}-l_{o}^{\mathrm{ss}}\right)\right] \approx \operatorname{Ei}\left(-\gamma l_{o}^{\mathrm{ss}}\right)-\left(l_{o} / l_{o}^{\text {ss }}\right) e^{-\gamma l_{o}^{\mathrm{ss}}}+O\left(l_{o}^{2}\right)$ to give a linear force-generating regime:

$$
l_{o} \approx \frac{\epsilon}{\left(1+v e^{-\epsilon / k_{b} T}\right)} \frac{1}{2 \Delta \eta} t .
$$

Thus, the predicted sliding velocity of the filaments is $V_{o}^{\mathrm{cl}}=\left(\epsilon P_{o}^{\mathrm{cl}} / 2 \Delta \eta\right)$ in the absence of kinetic arrest. In the long-time limit we can expand around $l_{o}=l_{o}^{\text {ss }}$ such that approximately $\operatorname{Ei}\left[\gamma\left(l_{o}-l_{o}^{\text {ss }}\right)\right] \approx \ln \left[\gamma\left(l_{o}^{\mathrm{ss}}-l_{o}\right)\right]$. This immediately gives an exponentially decaying overlap function to steady state:

$$
l_{o} \approx l_{o}^{\mathrm{ss}}-\left[\frac{1}{\gamma} \operatorname{Ei}\left(\gamma l_{o}^{\mathrm{ss}}\right)\right] e^{-b c^{-l \mathrm{ss} / \Delta} t},
$$

where the time scale of approaching steady state is given as $1 / \tau_{\mathrm{ss}}=b c^{-l_{o}^{\mathrm{ss}} / \Delta}=\left(K_{t} / \eta\right)\left(1+v^{-1} e^{\epsilon / k_{b} T}\right)^{-l_{o}^{\mathrm{ss}} / \Delta}$. To differentiate between the two power-law regimes, a transition time $\tau_{\text {trans }}$ is chosen when the slope of the time series in $l_{o}$ deviates by $10 \%$ for each cross-linker energy $\epsilon$.

\section{APPENDIX C: KINETIC BEHAVIOR OF THE ACTIVE FORCE DIPOLE}

We can derive the governing equation of the dynamics of the AFD from (un)binding kinetics of the passive and active components. We use the simple kinematics of a contraction event, $\xi_{0}\left(l_{o}, \tau\right)=$ $l_{o}\left(1-e^{\left(-K_{t} / 2 \eta\right) \tau}\right)$, and an extension event, $\chi_{0}\left(l_{o}, l_{w}, \tau\right)=$ $\left[\left(K_{m} / K_{\text {eff }}\right)\left(l_{o}+l_{w}\right)-l_{o}\right]\left(1-e^{\left(-K_{\text {eff }} / 2 \eta\right) \tau}\right)$, that come directly from solving the overlap relaxation of the element when a motor is (un)bound $(m=0,1)$ and cross-linkers are unbound $\left(n=0\right.$.) We have defined $K_{\text {eff }}=K_{m}+K_{t}$. If we are considering a cross-linker binding reaction with rate constant $k_{r}$ in our system which stops the contraction or extension motion, we must average $\chi$ and $\xi$ over the possible holding times of that reaction, $P\left(\tau \mid k_{r}\right)=k_{r} e^{-k_{r} \tau}$. Evaluating $\bar{\xi}\left(l_{o}\right)=\int_{0}^{\infty} \xi_{0}\left(l_{o}, \tau\right) P\left(\tau \mid k_{r}\right) d \tau$ and $\bar{\chi}\left(l_{o}, l_{w}\right)=$ $\int_{0}^{\infty} \chi_{0}\left(l_{o}, l_{w}, \tau\right) P\left(\tau \mid k_{r}\right) d \tau$, we have

$$
\bar{\xi}\left(l_{o}, k_{r}\right)=l_{o}\left(1+\frac{2 \eta k_{r}}{K_{t}}\right)^{-1},
$$

and 
$\bar{\chi}\left(l_{o}, l_{w}, k_{r}\right)=\left(\frac{K_{m}}{K_{\text {eff }}}\left(l_{o}+l_{w}\right)-l_{o}\right)\left(1+\frac{2 \eta k_{r}}{K_{\text {eff }}}\right)^{-1}$.

We consider the motor filament walking in the $n \neq 0$ state such that $F_{m} \approx K_{m} l_{w}$.

To write full expressions for $\chi\left(l_{o}\right)$ and $\xi\left(l_{o}\right)$ as observed in simulation, we in theory must consider all possible series of reaction events and their probabilities when a contraction or extension event takes place. However, to capture the average behavior of the element when $l_{o}$ is large and cross-link binding is sufficiently fast, the expected value of contraction, given an initial overlap $l_{o}$ can be written as $\mathbb{E}\left[\chi \mid l_{o}\right] \approx \bar{\chi}\left(l_{o}, k_{b}^{\mathrm{cl}}\right)$. The various pathways in which the motor filament can become (un)bound before cross-linkers rebind are insignificant to the mean behavior of the element in this case. The frequency of these contraction events is simply $\omega_{\chi}\left(l_{o}\right) \approx \rho_{m}\left[\bar{\tau}_{b}^{\mathrm{cl}}\left(l_{o}\right)+\right.$ $\left.\bar{\tau}_{u}^{\mathrm{cl}}\left(l_{o}\right)\right]^{-1}$, where we have defined the motor duty ratio in the zero-force limit: $\rho_{m}=k_{u}^{m} /\left(k_{u}^{m}+k_{b}^{m}\right)$. In a similar manner, the expected value of extension given an initial overlap can be written in a first-moment approximation as $\mathbb{E}\left[\xi \mid l_{o}\right] \approx \bar{\xi}\left(l_{o}, k_{b}^{\mathrm{cl}}\right)$ with a frequency $\omega_{\xi}\left(l_{o}\right) \approx$ $\left(1-\rho_{m}\right)\left[\bar{\tau}_{b}^{\mathrm{cl}}\left(l_{o}\right)+\bar{\tau}_{u}^{\mathrm{cl}}\left(l_{o}\right)\right]^{-1}$. These functions are plotted in Fig. 6 and agree well with collected data from Langevin simulations. The steady-state solution for $l_{o}$ by solving $V_{\text {eff }}=0$, i.e., $\rho_{m} \bar{\chi}\left(l_{o}\right)=\left(1-\rho_{m}\right) \bar{\xi}\left(l_{o}\right)$, is not analytically tractable.

\section{APPENDIX D: MEDYAN SIMULATION DETAILS}

To simulate realistic actin filaments interacting with transient cross-linking and motor filament molecules, the MEDYAN model solves a 3D stochastic reaction-diffusion master equation using a spatially resolved Gillespie algorithm $[53,54]$ with actin polymers imbedded in a reactiondiffusion simulation space. The simulated volume is divided into compartments with dimension based on the system's Kuramoto length [55]:

$$
l_{K}=\sqrt{6 D \tau}
$$

where $\tau$ represents the time scale of the fastest chemical reaction in our network (since this is cross-linker binding in most cases, this bounds $\tau \geq 1 \mathrm{~ms}$ and justifies $l_{K}=$ $500 \mathrm{~nm}$ in simulation). Diffusion of cross-linkers and motor filaments are modeled as jumps between uniformly spaced and assumed to be well-mixed compartments with reaction constant $D / l_{K}^{2}$.

The MEDYAN model also includes a mechanical representation of the disordered actin filament network which uses a cylinder-based scheme for semiflexible filaments with a persistence length, denoted as $l_{p}$, that is much larger than its diameter $\sigma_{0}$ (i.e., very large aspect ratio, $l_{p} \gg \sigma_{0}$ ). Filaments are divided into cylindrical segments of length $l_{c}=108 \mathrm{~nm}$. To account for the thermal elasticity of the filament segments, we use an angular potential between consecutive cylinders in the filament chain, written as

$$
U_{i}^{\text {bend }}=\varepsilon_{\text {bend }}\left[1-\cos \left(\theta_{i, i+1}\right)\right],
$$

where $\theta_{i, i+1}$ is the angle between two consecutive cylinders $i$ and $i+1$ along the filament chain, and we use $\varepsilon_{\text {bend }}=k_{b} T l_{c} / l_{p}$, where $l_{p} \approx 17 \mu \mathrm{m}$ for an actin filament [50]. We can also introduce a stretching potential of a cylinder along its main axis, representing elastic deformations of the filament beyond its entropically driven elastic limit [56]. This is written as

$$
U_{i}^{\mathrm{str}}=\frac{1}{2} K_{\mathrm{str}}\left(\left|\boldsymbol{l}_{i}\right|-l_{c}\right)^{2},
$$

where $\boldsymbol{l}_{i}=\mathbf{x}_{i}^{2}-\mathbf{x}_{i}^{1}$ is the vector connecting the end points of the $i$ th cylinder. We assume an extremely stiff actin stretching constant of $K_{\text {str }}=100 \mathrm{pN} / \mathrm{nm}$.

Cross-linkers and motor filaments can bind onto neighboring cylinders $i$ and $j$ at the positions $\mathbf{x}_{i}$ and $\mathbf{x}_{j}$ and create a mechanical bond. To describe the stretching energy of a bond created by a motor filament or cross-linker, we use a simple harmonic potential:

$$
U_{i j}^{\text {bond }}=\frac{1}{2} K_{m, \mathrm{cl}}\left(\left|\boldsymbol{l}_{i j}\right|-l_{0}\right)^{2},
$$

where $\boldsymbol{l}_{i j}=\mathbf{x}_{j}-\mathbf{x}_{i}$ is the instantaneous length of the motor or cross-linker, $l_{0}$ is the equilibrium length of the particular motor or cross-linker $(35 \pm 5$ or $200 \pm 20 \mathrm{~nm}$ upon binding, respectively), and $K_{\text {str }}$ is the stretching constant of the motor filament or cross-linker $\left(K_{\mathrm{ms}}=0.5 \mathrm{pN} / \mathrm{nm}\right.$ [7] and $K_{\mathrm{cl}}=8 \mathrm{pN} / \mathrm{nm}$ [41], respectively). In the case of molecular motors, the binding position of the motor head can change stochastically due to a chemical reaction, producing forces on the bound filament. The stretching constant of a motor filament $K_{m}$ is updated based on the number of bound heads to actin from the total ensemble containing $N_{t}$ heads, as elaborated in Appendix A.

In order to describe a rigorous excluded volume interaction between two cylindrical units on neighboring filaments to disallow the crossing of the segments, denoted as $i$ and $j$, we introduce the following potential:

$$
U_{i j}^{\mathrm{vol}}=\iint_{l_{i}, l_{j}} \delta U\left(\left|\mathbf{r}_{i}-\mathbf{r}_{j}\right|\right) d l_{i} d l_{j} .
$$

Here, $\delta U\left(\left|\mathbf{r}_{i}-\mathbf{r}_{j}\right|\right)$ is a pair potential between two points located on the two interacting cylinders $i$ and $j$. For purely excluded volume repulsion, we have chosen $\delta U\left(\left|\mathbf{r}_{i}-\mathbf{r}_{j}\right|\right)=$ $K_{\text {vol }} /\left|\mathbf{r}_{i}-\mathbf{r}_{j}\right|^{4}$. This provides a steep enough function to mimic cylindrical hard-core repulsion, while allowing the line integrals defined previously to be evaluated analytically.

Lastly, filament plus ends $\mathbf{x}_{i}^{p}$ are tethered to initial positions $\mathbf{x}_{i}^{t}$ by a harmonic potential: 


$$
U_{i}^{\text {tether }}=\frac{1}{2} K_{t}\left(\left|\mathbf{x}_{i}^{p}-\mathbf{x}_{i}^{t}\right|\right)^{2} .
$$

The total of all potentials $U^{\text {tot }}$ is used to mechanically equilibrate the network after a number of chemical steps evolve the reactive elements in time, producing mechanical response to network growth and deformation. This is possible due to the separation of time scales for chemical kinetics $\left(\tau_{k} \approx \mathrm{ms}\right)$ and local mechanical relaxation of active stress $\left(\tau_{r} \approx \mu\right.$ s [36]), i.e., $\tau_{k} \gg \tau_{r}$. Networks are mechanically equilibrated using a conjugate gradient minimization algorithm every $0.05 \mathrm{~s}$ of stochastic chemical events.

[1] B. Alberts, A. Johnson, J. Lewis, M. Raff, K. Roberts, and P. Walter, Molecular Biology of the Cell, 4th ed. (Garland Science, New York, 2002).

[2] A. Schmidt and M. N. Hall, Signaling to the Actin Cytoskeleton, Annu. Rev. Cell Dev. Biol. 14, 305 (1998).

[3] V. Vogel and M. Sheetz, Local Force and Geometry Sensing Regulate Cell Functions, Nat. Rev. Mol. Cell Biol. 7, 265 (2006).

[4] T. Luo, K. Mohan, P. A. Iglesias, and D. N. Robinson, Molecular Mechanisms of Cellular Mechanosensing, Nat. Mater. 12, 1064 (2013).

[5] A. V. Hill, The Mechanical Eefficiency of Frog's Muscle, Proc. R. Soc. B 127, 434 (1939).

[6] T. Duke, Molecular Model of Muscle Contraction, Proc. Natl. Acad. Sci. U.S.A. 96, 2770 (1999).

[7] A. Vilfan and T. Duke, Instabilities in the Transient Response of Muscle, Biophys. J. 85, 818 (2003).

[8] N. Billington, A. Wang, J. Mao, R. S. Adelstein, and J. R. Sellers, Characterization of Three Full-Length Human Nonmuscle Myosin II Paralogs, J. Biol. Chem. 288, 33398 (2013).

[9] G. Salbreux, G. Charras, and E. Paluch, Actin Cortex Mechanics and Cellular Morphogenesis, Trends Cell Biol. 22, 536 (2012).

[10] R. Levayer and T. Lecuit, Biomechanical Regulation of Contractility: Spatial Control and Dynamics, Trends Cell Biol. 22, 61 (2012).

[11] L. Blanchoin, R. Boujemaa-Paterski, C. Sykes, and J. Plastino, Actin Dynamics, Architecture, and Mechanics in Cell Motility, Physiol. Rev. 94, 235 (2014).

[12] M. Lenz, Geometrical Origins of Contractility in Disordered Actomyosin Networks, Phys. Rev. X 4, 041002 (2014).

[13] M. Lenz, M. L. Gardel, and A. R. Dinner, Requirements for Contractility in Disordered Cytoskeletal Bundles, New J. Phys. 14, 033037 (2012).

[14] M.P. Murrell and M. L. Gardel, F-Actin Buckling Coordinates Contractility and Severing in a Biomimetic Actomyosin Cortex, Proc. Natl. Acad. Sci. U.S.A. 109, 20820 (2012).

[15] M. S. e Silva, M. Depken, B. Stuhrmann, M. Korsten, F. C. MacKintosh, and G. H. Koenderink, Active Multistage Coarsening of Actin Networks Driven by Myosin Motors, Proc. Natl. Acad. Sci. U.S.A. 108, 9408 (2011).
[16] D. B. Oelz, B. Y. Rubinstein, and A. Mogilner, A Combination of Actin Treadmilling and Cross-Linking Drives Contraction of Random Actomyosin Arrays, Biophys. J. 109, 1818 (2015).

[17] K. Popov, J. Komianos, and G. A. Papoian, MEDYAN: Mechanochemical Simulations of Contraction and Polarity Alignment in Actomyosin Networks, PLoS Comput. Biol. 12, e1004877 (2016).

[18] P. M. Bendix, G. H. Koenderink, D. Cuvelier, Z. Dogic, B. N. Koeleman, W. M. Brieher, C. M. Field, L. Mahadevan, and D. A. Weitz, A Quantitative Analysis of Contractility in Active Cytoskeletal Protein Networks, Biophys. J. 94, 3126 (2008).

[19] S. Köhler and A. R. Bausch, Contraction Mechanisms in Composite Active Actin Networks, PLoS One 7, 1 (2012).

[20] S. Wang and P. G. Wolynes, Active Contractility in Actomyosin Networks, Proc. Natl. Acad. Sci. U.S.A. 109, 6446 (2012).

[21] J. Alvarado, M. Sheinman, A. Sharma, F. C. MacKintosh, and G. H. Koenderink, Molecular Motors Robustly Drive Active Gels to a Critically Connected State, Nat. Phys. 9, 591 (2013).

[22] W. Jung, M. P. Murrell, and T. Kim, F-Actin Cross-Linking Enhances the Stability of Force Generation in Disordered Actomyosin Networks, Comput. Part. Mech. 2, 317 (2015).

[23] H. Ennomani, G. Letort, C. Guérin, J.-L. Martiel, W. Cao, F. Nédélec, E. M. De La Cruz, M. Théry, and L. Blanchoin, Architecture and Connectivity Govern Actin Network Contractility, Curr. Biol. 26, 616 (2016).

[24] S. Wang and P. G. Wolynes, Communication: Effective Temperature and Glassy Dynamics of Active Matter, J. Chem. Phys. 135, 051101 (2011).

[25] A. J. Levine and F. C. MacKintosh, The Mechanics and Fluctuation Spectrum of Active Gels, J. Phys. Chem. B 113, 3820 (2009).

[26] S. Walcott and S.X. Sun, Active Force Generation in Cross-Linked Filament Bundles without Motor Proteins, Phys. Rev. E 82, 050901 (2010).

[27] Z. Lansky, M. Braun, A. Lüdecke, M. Schlierf, P. R. T. Wolde, M. E. Janson, and S. Diez, Diffusible Crosslinkers Generate Directed Forces in Microtubule Networks, Cell 160, 1159 (2015).

[28] J. Hermans and B. Lentz, Equilibria and Kinetics of Biological Macromolecules (Wiley, New York, 2014).

[29] T. D. Pollard, Structure and Polymerization of Acanthamoeba Myosin-II Filaments, J. Cell Biol. 95, 816 (1982).

[30] S. X. Sun, S. Walcott, and C. W. Wolgemuth, Cytoskeletal Cross-Linking and Bundling in Motor-Independent Contraction, Curr. Biol. 20, R649 (2010).

[31] D. Johann, D. Goswami, and K. Kruse, Generation of Stable Overlaps between Antiparallel Filaments, Phys. Rev. Lett. 115, 118103 (2015).

[32] T. Erdmann, P. J. Albert, and U.S. Schwarz, Stochastic Dynamics of Small Ensembles of Non-Processive Molecular Motors: The Parallel Cluster Model, J. Chem. Phys. 139, 175104 (2013).

[33] S. Stam, J. Alberts, M. L. Gardel, and E. Munro, Isoforms Confer Characteristic Force Generation and Mechanosensation by Myosin II Filaments, Biophys. J. 108, 1997 (2015). 
[34] Assuming a critical buckling force for a cross-linked segment of the form $f_{c} \approx \pi^{2} k_{b} T l_{p} / L^{2}$, where $l_{p}=17 \mu \mathrm{m}$ for an actin filament [35], and the inter-cross-link distance of the actin filament network being $L=$ $0.4 \mu \mathrm{m}$ [36], which is representative of lamellar actin concentrations of $30 \mu \mathrm{M}$, the estimated critical buckling force for these network segments is $4.3 \mathrm{pN}$. Assuming ten motor heads of nonmuscle myosin II (isoform A) are available for binding to an actin segment, predictions for force generation of this small ensemble are well below $5 \mathrm{pN}$ due to the stochastic nature of the nonprocessive motor heads $[32,33]$. We also confirm this in our force dipole simulations.

[35] M. Footer, J. Kerssemakers, J. Theriot, and M. Dogterom, Direct Measurement of Force Generation by Actin Filament Polymerization Using an Optical Trap, Proc. Natl. Acad. Sci. U.S.A. 104, 2181 (2007).

[36] T. T. Falzone, S. Blair, and R. M. Robertson-Anderson, Entangled F-Actin Displays a Unique Crossover to Microscale Nonlinearity Dominated by Entanglement Segment Dynamics, Soft Matter 11, 4418 (2015).

[37] Y. Lan and G. A. Papoian, The Stochastic Dynamics of Filopodial Growth, Biophys. J. 94, 3839 (2008).

[38] P. I. Zhuravlev and G. A. Papoian, Molecular Noise of Capping Protein Binding Induces Macroscopic Instability in Filopodial Dynamics. Proc. Natl. Acad. Sci. U.S.A. 106, 11570 (2009).

[39] L. Hu and G. A. Papoian, Mechano-Chemical Feedbacks Regulate Actin Mesh Growth in Lamellipodial Protrusions, Biophys. J. 98, 1375 (2010).

[40] L. Hu and G. A. Papoian, Molecular Transport Modulates the Adaptive Response of Branched Actin Networks to an External Force, J. Phys. Chem. B 117, 13388 (2013).

[41] J. M. Ferrer, H. Lee, J. Chen, B. Pelz, F. Nakamura, R. D. Kamm, and M.J. Lang, Measuring Molecular Rupture Forces between Single Actin Filaments and ActinBinding Proteins, Proc. Natl. Acad. Sci. U.S.A. 105, 9221 (2008).

[42] M. Murrell, P. W. Oakes, M. Lenz, and M. L. Gardel, Forcing Cells into Shape: The Mechanics of Actomyosin Contractility, Nat. Rev. Mol. Cell Biol. 16, 486 (2015).

[43] M. Kovács, K. Thirumurugan, P. J. Knight, and J. R. Sellers, Load-Dependent Mechanism of Nonmuscle Myosin 2. Proc. Natl. Acad. Sci. U.S.A. 104, 9994 (2007).
[44] B. Guo and W. H. Guilford, Mechanics of Actomyosin Bonds in Different Nucleotide States Are Tuned to Muscle Contraction, Proc. Natl. Acad. Sci. U.S.A. 103, 9844 (2006).

[45] S. Walcott, D. M. Warshaw, and E. P. Debold, Mechanical Coupling between Myosin Molecules Causes Differences between Ensemble and Single-Molecule Measurements, Biophys. J. 103, 501 (2012).

[46] C. Veigel, J. E. Molloy, S. Schmitz, and J. Kendrick-Jones, Load-Dependent Kinetics of Force Production by Smooth Muscle Myosin Measured with Optical Tweezers, Nat. Cell Biol. 5, 980 (2003).

[47] M. F. Norstrom, P. A. Smithback, and R. S. Rock, Unconventional Processive Mechanics of Non-Muscle Myosin IIB, J. Biol. Chem. 285, 26326 (2010).

[48] D. H. Wachsstock, W. H. Schwartz, and T. D. Pollard, Affinity of Alpha-Actinin for Actin Determines the Structure and Mechanical Properties of Actin Filament Gels, Biophys. J. 65, 205 (1993).

[49] X. Veigel, C. J. E. Molloy, and J. Kendrick-Jones, LoadDependent Kinetics of Force Production by Smooth Muscle Myosin Measured with Optical Tweezers, Nat. Cell Biol. 5, 980 (2003).

[50] A. Ott, M. Magnasco, A. Simon, and A. Libchaber, Persistence of Actin, Macromolecules 48, 1642 (1993).

[51] M. Kovács, F. Wang, A. Hu, Y. Zhang, and J. R. Sellers, Functional Divergence of Human Cytoplasmic Myosin II. Kinetic Characterization of the Non-Muscle IIA Isoform, J. Biol. Chem. 278, 38132 (2003).

[52] G. Piazzesi, M. Reconditi, M. Linari, L. Lucii, P. Bianco, E. Brunello, V. Decostre, A. Stewart, D. B. Gore, T. C. Irving, M. Irving, and V. Lombardi, Skeletal Muscle Performance Determined by Modulation of Number of Myosin Motors Rather than Motor Force or Stroke Size, Cell 131, 784 (2007).

[53] D. T. Gillesple, Exact Stochastic Simulation of Coupled Chemical Reactions, J. Phys. Chem. 93555, 2340 (1977).

[54] D. T. Gillespie, A General Method for Numerically Simulating the Stochastic Time Evolution of Coupled Chemical Reactions, J. Comput. Phys. 22, 403 (1976).

[55] Y. Kuramoto, Effects of Diffusion on the Fluctuations in Open Chemical Systems, Prog. Theor. Phys. 52, 711 (1974).

[56] F. C. MacKintosh, Polymer-Based Models of Cytoskeletal Networks, Cytoskelet. Mech. Model. Meas. Cell Mech. 2006, 152 (2006). 